\title{
Zwischen Kundenakquise und gelehrter Windbeuteley
}

\author{
Christian Thomasius' frühe akademische Programmschriften \\ im Kontext zeitgenössischer Praktiken der Vorlesungs- \\ ankündigung
}

\section{Einleitung}

Die Erzählung vom universitären Sprachwechsel ist im Kern eine heroische. Sie bedient sich einer von den Zeitgenossen zunächst wenig beachteten, jedoch früh zum Heldenplot umgeformten Begebenheit des Jahres 1687 (vgl. Prinz 2019), deren narrative Modellierung sich in der akademischen Domäne bis in die „postheroische Gegenwart“ (Münkler 2015: Teil II) hinein als wirkmächtig erweist: Christian Thomasius' Publikation einer volkssprachig-deutschen Vorlesungseinladung am schwarzen Brett der Universität Leipzig:

\begin{abstract}
Als ich für ohngefehr dreyßig Jahren ein teutsch Programma in Leipzig an das schwartze Bret schlug/ in welchem ich andeutete/ daß ich über des Gracians Homme de cour lesen wolte/ was ware da nicht für ein entsetzliches lamentiren! Denckt doch! ein teutsch Programma an das lateinische schwartze Bret der löbl. Universität. Ein solcher Greuel ist nicht erhöret worden/ weil die Universität gestanden. Ich muste damahls in Gefahr stehen/ daß man nicht gar solenni processione das löbliche schwartze Bret mit Weyhwasser besprengte. (Thomasius 1717: 252)
\end{abstract}

Die gängige Bedeutungszuschreibung für diese grenzüberschreitende Handlung erhellt bereits aus wenigen Zitaten: Ein „unerhörter Vorgang“ (StollbergRilinger 2000: 19) sei der „spektakulär inszenierte Sprachenwechsel“ gewesen (Polenz 1994: 55), eine „Luthertat“ (Fleischmann 1929: 7), mit der der junge Jurist „die Thesen zur Reformation der deutschen Wissenschaft an das schwarze Brett“" geschlagen habe (Hodermann 1891: 39). Die sozialsymbolische Bedeutung der Sprachwahl als bewusste Abkehr von der gelehrten lateinischen Wissenschaftstradition erhebe den Leipziger Thesenanschlag zu einem Ereignis von enormer wissenschaftsgeschichtlicher Tragweite und Bedeutsamkeit. Entsprechend erscheint bis heute kaum eine Publikation zur Geschichte des Deut-

Michael Prinz, Universität Zürich, Deutsches Seminar, Schönberggasse 9, CH-8001 Zürich, E-Mail: michael.prinz@ds.uzh.ch

Ә Open Access. (C) 2018 Michael Prinz, publiziert von De Gruyter. (c) BY-NC-ND Dieses Werk ist lizenziert unter der Creative Commons Attribution-NonCommercial-NoDerivatives 4.0 Lizenz. 
schen als Wissenschaftssprache - im esoterischen wie im exoterischen Kreis der an historischer Wissenschaftskommunikation Interessierten -, die nicht an irgendeiner Stelle das schwarze Brett der Leipziger Universität als obligatorischen Erinnerungsort aufsuchen würde. Angesichts der Persistenz dieser Heldenerzählung erstaunt es, dass zu diesem für die historische Selbstvergewisserung deutschsprachiger Wissenschaftskulturen so bedeutsamen Vorgang zahlreiche Ungereimtheiten in Umlauf sind. Ziel des Beitrags ist es deshalb, die genauen Umstände von Thomasius' Ankündigung zu rekonstruieren und dabei insbesondere die zeitgenössischen Medien der Vorlesungseinladung in den Blick zu nehmen.

\section{Historische Vorlesungsankündigungen}

In jüngerer Zeit sind historische Medien der Vorlesungsankündigung verstärkt ins Blickfeld der wissenschafts- und universitätsgeschichtlichen Forschung gerückt. ${ }^{1}$ Oft werden dabei unter der modernen ${ }^{2}$ Bezeichnung Vorlesungsverzeichnis (im weiteren Sinne) verschiedene vorlesungsbezogene Quellengattungen bzw. Textsorten subsumiert, deren Wurzeln meist ins 16. Jahrhundert zurückreichen (vgl. Rasche 2009a: 447-449; Bruning 2011: 270-276):

(I.) Periodische Lektionskataloge (Catalogi Praelectionum) bzw. Vorlesungsverzeichnisse im engeren Sinne entstanden ursprünglich wohl aus nur sporadisch erscheinenden Lektionsplänen (dazu Rasche 2009a: 449 f.). Unter einem Lektionskatalog wird dabei eine offizielle Aufstellung des universitären oder fakultären ${ }^{3}$ Lehrkursus bzw. Lehrangebots für ein bestimmtes Semester

1 Vgl. etwa Schneider (1999: 60-75), Pozzo (1999), Clark (2006: 33-67), Rasche (2009a), Bruning (2011), Pozzo (2012) und Le Cam (2016). Einzeluntersuchungen liegen mittlerweile für eine Reihe von Universitäten vor, etwa für Helmstedt (Bruning 2012: 70-85), Jena (Bach, Maatsch \& Rasche 2008), Königsberg (Oberhausen \& Pozzo 1999) und Leipzig (Huttner 2007a bzw. - weitgehend textidentisch - 2007b: 177-199; daneben auch Döring 2009: 678-680). In der Folge liegt der Fokus v. a. auf den Verhältnissen in Leipzig und an den dafür besonders relevanten omd. bzw. preuss. Nachbaruniversitäten.

2 DWDS (www.dwds.de) und Google Books liefern für das Wort Belege seit etwa 1870 (01. 02. 2018).

3 So enthält etwa der Codex Lectionum der Universität Halle spezielle Lektionskataloge der Theologischen Fakultät für folgende Semester: Sommersemester 1697, Wintersemester 1709/ 1719, Sommersemester 1712, Sommersemester 1717 (Halle, Universitäts- und Landesbibliothek, Yb 3885c, 2 , Bl. 7, 32, 48, 64). Das bei Blanke (1988: 112) erwähnte „Vorlesungsverzeichnis der Philosophischen Fakultät WS 1715/16“ ist dagegen in Wirklichkeit nur der bei Paulsen (1919: 635 f.) unter dem irreführenden Titel index scholarum gedruckte Abschnitt aus dem regulären 
oder Studienjahr verstanden, welche regelmäßig als Separatdruck universitätsöffentlich, d.h. am Schwarzen Brett, bekannt gegeben wurde. Zunächst enthielten diese Texte nur die öffentlichen Vorlesungen (lectiones publicae) der im Katalog nach Rang bzw. Anciennität ${ }^{4}$ abfolgenden Professoren, später dann auch deren Privatlehre. Im 18. Jahrhundert durchliefen die Lektionskataloge einen mehrstufigen Transformationsprozess (vgl. Blanke 1983: 205 f. u. 1987: $18 \mathrm{f}$.): Ab den 1720er Jahren und vor allem in der zweiten Jahrhunderthälfte begannen Universitäten, zusätzlich zu den lateinischen Katalogen zunehmend auch deutsche Fassungen für eine außeruniversitäre Öffentlichkeit zu publizieren. Über Intelligenzblätter und gelehrte Journale, die häufig einen Bezug zur jeweiligen Universität hatten (vgl. Huttner 2007a: 194), wurde das akademische Schwarze Brett in die weite Welt der res publica litteraria hinausprojiziert, in dem selbstbewussten Glauben, „dass vielen exteris dadurch ein grosser Dienst“ ${ }^{5}$ erwiesen werde. Später erschien die deutsche Fassung dann auch als Separatdruck parallel zur lateinischen.

Heutige universitäre Vorlesungsverzeichnisse erscheinen als eine orientierende assertive Gebrauchstextsorte. ${ }^{6}$ Natürlich erlaubten auch die Lektionspläne und -kataloge der Frühen Neuzeit durch die strukturierte Anordnung der einzelnen Einträge und die reduzierte, lediglich indizierende Präsentation der jeweiligen Lehrveranstaltungen einen schnellen Zugriff auf relevante Informationen. Stärker als heute lassen die vormodernen Verzeichnisse neben der Informationsfunktion jedoch auch direktive Züge erkennen. Die universitätsgeschichtliche Forschung ${ }^{7}$ hat gerade in jüngerer Zeit die Polyfunktionalität historischer Vorlesungsverzeichnisse betont: als beständige Ermahnung der Studenten im Hinblick auf den tradierten Lehrkursus, als Mittel der Disziplinierung von Professoren, vor allem aber als Marketing- und Repräsentationsins-

Lektionskatalog (Bl. 58). In Leipzig dagegen haben sich zwischen 1641 und 1680 tatsächlich insgesamt 18 Kataloge der Philosophischen Fakultät erhalten (vgl. Huttner 2007a: 192).

4 Entsprechend der Auflistung bei Clark (2006: 479 Table 2) erfolgte die Umstellung auf eine (partielle oder vollständige) disziplinäre Anordnung der Lehrveranstaltungen im Katalog an den meisten Universitäten des deutschsprachigen Raums erst ab der zweiten Hälfte des 18. Jahrhunderts.

5 So anlässlich der Bekanntgabe der Leipziger Publica durch den Rektor im fünften Teil der Acta Lipsiensium Academica (1723: 460 Anm. a). Die Herausgeber (zur Herausgeberfrage vgl. Döring 2002: 143 Anm. 92) fühlten sich allerdings zu der Rechtfertigung verpflichtet, sie würden die öffentlichen Lektionen „Lateinisch so, wie sie publiciret worden, communiciren, weil wir, der vielen Lateinischen terminorum wegen, der teutschen Sprache mit der Ubersetzung keine Gewalt anthun wollen.“

6 Sie entsprechen damit dem Typ „ASS 38“ (Assertiva) der Klassifikation von Rolf (1993).

7 Vgl. dazu etwa Huttner (2007a: 198) und Rasche (2009a: 453-458 u. 463-465). 
trument der Universität - ein Aspekt, der bereits den Schriftsteller Arnold Ruge (1840: 1905) zu der spitzen Bemerkung veranlasste, solche Texte seien „weniger dazu bestimmt, gelesen als vielmehr nur geschrieben zu werden“. Die Universitäten befanden sich zunehmend in einer Konkurrenzsituation, ${ }^{8}$ die es lohnend erscheinen ließ, sich einer überregionalen Öffentlichkeit durch die publizistische Verbreitung der eigenen Lektionskataloge als attraktiver Studienort zu präsentieren. Während aber mit der Auflösung des traditionellen ordo lectionum die beiden erstgenannten Funktionen im Laufe des 18. Jahrhunderts verloren gingen, wurden die Lektionskataloge zunehmend wichtig für die praktische Semesterplanung. Rasche (2009a: 466-469) gelangt sogar zu der überraschenden Einschätzung, dass damit die konkrete Studieninformation als eine „neue Funktion“ neben die alte Funktion der Universitätswerbung getreten sei. Mindestens subsidiär dürften die Vorlesungsverzeichnisse aber wohl auch vorher schon einen orientierenden und damit assertiven Charakter gehabt haben. ${ }^{9}$

Vor diesem Hintergrund ist es allerdings schwierig, eine über die gesamte Existenzdauer der Vorlesungsverzeichnisse (i.e.S.) stabile textuelle Grundfunktion zu benennen. Selbst für das 18. Jahrhundert zeichnet sich prima facie nicht eine dominierende Textfunktion ab. ${ }^{10}$ So teilte etwa der Rektor der Universität Halle der Studentenschaft („civibus academicis“) ${ }^{11}$ die Veranstaltungen des Folgesemesters am Anfang des Codex Lectionum (s. Anm. 3) stets mit der Formel „publicat et commendat“ mit. Und auch sein Leipziger Kollege vermeldete und empfahl - „solemniter indicit et diligenter commendat“ - die dortigen Vorlesungen in einem Atemzug (vgl. das Faksimile bei Döring 2009: 679). Die Verzeichnisse drücken somit eine doppelte Handlungsabsicht aus und signalisieren dem Adressaten ihre Informations- wie auch Appellfunktion direkt. Es wäre eine lohnende Aufgabe zukünftiger textlinguistischer Forschung, das

\footnotetext{
8 In Leipzig entstand eine solche Konkurrenz v. a. durch die Universitätsneugründungen in Halle und Göttingen (dazu zuletzt Pohl 2016: 98-100).

9 Es finden sich durchaus Indizien für die Nützlichkeit der Lektionskataloge als Informationsquelle für die Stundenplanung der Studenten. So weisen z. B. die Kataloge der Leipziger Philosophischen Fakultät (s. Anm. 3) bereits Mitte des 17. Jahrhunderts eine chronologische Strukturierung entsprechend der reservierten professoralen Vorlesungszeitfenster auf und nicht die sonst übliche hierarchische Gliederung nach akademischem „Charisma“ (vgl. dazu Huttner 2007a: 192).
}

10 Dies ist freilich nur ein Problem, wenn man (wie Rolf 1993: 39-43 u. 91 oder Brinker 2000: 176 f.) eine Position weitgehender Monofunktionalität einnimmt, die allerdings „oft nicht beschreibungsadäquat“ erscheint (Fandrych \& Thurmair 2011: 20). Zur umfangreichen Diskussion über Mono- oder Polyfunktionalität von Textsorten vgl. etwa Rolf (2000), Heinemann (2008: 130 f.), Adamzik (2016: 178-182 u. 196-198) und Hausendorf et al. (2017: 236-244).

11 Zum Ausdruck civis academicus vgl. etwa Gundling (1744: 1406) und [Anonym] (1751: 712). 
Gefüge von dominierenden und subsidiären Textfunktionen in den historischen Vorlesungen auf einer breiten Textgrundlage zu erfassen und die angedeuteten diachronen Verschiebungen präzise zu rekonstruieren.

(II.) Die bislang wenig erforschten Rechenschaftsberichte der Professoren über die von ihnen gehaltenen Vorlesungen, mitunter etwas missverständlich ${ }^{12}$ als Vorlesungszettel oder Lektionszettel bezeichnet, gewannen im Zuge des Ausbaus der landesherrlichen Aufsicht über die Universitäten zunehmend an Bedeutung (vgl. Clark 2006: 48-52). Als eine registrierende Textsorte dienen die Rechenschaftsberichte dazu, ein vergangenes Geschehen akkurat zu erfassen, konkret: „über die Art und/oder die Ergebnisse eines [...] selbst realisierten Komplexes von Tätigkeiten“ Aufschluss zu geben. ${ }^{13}$

(III.) Noch unmittelbarer als beim ersten Typ handelt es sich bei akademischen Programmschriften (Programmata) ${ }^{14}$ um eine Textsorte mit dominierender Steuerungs- bzw. Appellfunktion. Mit dieser „weithin vergessenen Quellengattung“ (Marti 2013) bewarben Dozenten ihre eigenen künftigen Veranstaltungen, und zwar in Gestalt von Drucken unterschiedlichen Formats und Umfangs (von plakatartigen Einblattprogrammen im Folio-Format bis zu umfangreichen oktavformatigen Heften). ${ }^{15}$ Die Programmata lieferten, ähnlich wie später die kommentierten Vorlesungsverzeichnisse, mehr oder weniger ausführliche Informationen über Rahmenbedingungen und Inhalte der angekündigten Lehrveranstaltungen, wobei von einem nicht-bindenden Durchsetzungsmodus und einem beiderseitigen Interesse von Textproduzent und -addressat auszugehen ist. ${ }^{16}$ Während die offiziellen Lektionskataloge der Repräsentation und öffentlichen Inszenierung der Universität dienten, hatten die privaten Programm-

12 Beide Bezeichnungen kommen auch häufig für die Anschlagzettel des Typs (III.) vor, etwa bei Rüegg (1996: 270) oder Neuper (2003: v).

13 Rolf (1993: 186); solche Texte wären entsprechend in die Gruppe „ASS [= Assertiva] 12“ der Typologie von Rolf einzuordnen.

14 Als wissenschaftssprachlicher Terminus ist programma an deutschen Universitäten seit dem 17. Jh. in Gebrauch, und zwar für ,öffentliche Bekanntmachungen von Informationen über die akademisch-universitären Organisationsformen oder Veranstaltungen“, konkret für Einladungen zu Vorlesungen, öffentlichen Disputationen, Inauguralreden, akademischen Feiern, Totenehrungen etc., aber auch für Probevorlesungen (vgl. Neumann 2005: Sp. 155 f.). Zugleich dienten die Programmata als Nachweise für den Erwerb akademischer Titel (dazu Dinkel 2000: 428).

15 Die von Lee et al. (2012) ausgewählten akademischen Programmschriften der deutschen Aufklärung sind im Original zwischen 16 und 80 Seiten lang. Auch bei Thomasius' Programmata variiert die Länge erheblich: Während das Gracián-Programm VE 5 von 1687 immerhin 40 Seiten (38 S., [1] Bl.) in $4^{\circ}$ umfasst, ist das im selben Jahr gedruckte lateinische Programm VE 4 lediglich 4 Seiten ([2] Bl.) in $2^{\circ}$ lang.

16 Sie entsprechen damit Gruppe 13 der Direktiva bei Rolf (1993). 
schriften der Dozenten einen völlig anderen Charakter: „[T]heir contents were enticing and hyperbolic, given the professor's aim of gaining the benevolence of the students by condescending to their reasoning abilities“ (Pozzo 2012: IX). Da die Programmata über die eigentliche Lehrankündigung hinaus noch umfangreiche Einlassungen zu wissenschaftlichen Fragestellungen enthalten konnten, behielten sie mitunter auch außerhalb ihres ursprünglichen Funktionszusammenhangs einen gewissen thematischen Eigenwert, der zur Aufnahme in zeitgenössische Werkverzeichnisse führen und sogar einen separaten oder auch kompilatorischen Wiederabdruck rechtfertigen konnte. ${ }^{17}$

Die konkrete Einladung $\mathrm{zu}$ einer universitären Lehrveranstaltung am Schwarzen Brett erfolgte allerdings häufig mittels einfacher handschriftlicher Anschlagzettel (Schedae), die das gedruckte Informationsangebot präzisieren oder aktualisieren konnten ${ }^{18}$ und unter besonderen Umständen bis heute erhalten geblieben sein können, ${ }^{19}$ gerade bei juristischen Streitfällen. Beispielsweise entspinnt sich im Oktober/November 1675 ein Streit zwischen der Leipziger Juristenfakultät und einem Dr. Gottfried Kellner, der „des öffentlichen Anschlagens sich eigenthätiger Weise angemaßet“ habe. In den Rektoratsakten $\mathrm{zu}$ diesem Vorgang findet sich noch der handschriftliche lateinische Anschlagzettel mit Befestigungslöchern, also das auf Anweisung des Rektors abgenommene Blatt. ${ }^{20}$ Im Verlauf des 18. Jahrhunderts verdrängen dann die Anschlagzettel, welche z.B. in Jena ab der Jahrhundertmitte zunehmend auf Deutsch verfasst wurden, die akademischen Programmschriften (vgl. Rasche 2008: $32 \mathrm{f}$.).

Programmschriften und Anschlagzettel können zusammenfassend als Vorlesungseinladungen bezeichnet werden (dazu Anm. 51). Da sie die individuellen Lehrangebote eines einzelnen Textproduzenten dokumentieren, nicht die kollektive Lehre eines gesamten Lehrkörpers wie die Vorlesungsverzeichnisse,

17 Vgl. das aufschlussreiche Beispiel des Helmstedter Rhetorikprofessors Christoph Schrader, dem 1667 vom eigenen Sohn eine ehrende Kompilation der Programmata aus drei Jahrzehnten Universitätslehre herausgegeben wurde (dazu Le Cam 2016: 101 f.). Von Thomasius' frühen Programmschriften erfuhren VE 1, 2 und 5 einen separaten Wiederabdruck. Eine Gesamtausgabe der Programmata erfolgte dann - getrennt nach Sprache - im Rahmen von KTS (1701) und PT (1724).

18 So zeigen etwa die erhaltenen handschriftlichen Zettel zu den Jenaer Vorlesungen des Philosophen Christian Gottfried Schütz aus den 1780er Jahren häufig Veränderungen gegenüber dem im Lektionskatalog veröffentlichten Text (vgl. Schröpfer 2003: 84).

19 Eine systematische Sammlung solcher Texte, wie im Fall der Universität Jena (vgl. Rasche 2008: 29-37), dürfte die Ausnahme sein. Für Göttingen geht Tütken (2005: 7) jedenfalls davon aus, dass die Anschlagzettel der Privatdozenten „keine papierenen Spuren hinterlassen“ haben.

20 UAL, Rektor, Rep. 01/05/011, fol. 5r: „Acta H. D. Gottfried Kellners angeschlagene Collegia“. 
weisen sie eine größere Informationsfülle und eine stärkere Adressatenorientierung auf: Sie benennen die Inhalte der Veranstaltung, verorten diese ggf. in einem relevanten Fachdiskurs und spezifizieren die Modalitäten der Teilnahme (Termin, Gebühren, Lehrbuch etc.). Mitunter werden auch in längeren Exkursen zusätzliche Themen behandelt (vgl. Marti 2013).

Dabei sind Vertreter des Typs (I.) obligatorisch komplex - Vorlesungsverzeichnisse umfassen stets mehrere Veranstaltungen. Für die übrigen Typen ist dieses Merkmal definitorisch irrelevant, da Vorlesungseinladungen und Rechenschaftsberichte für einzelne wie für mehrere Veranstaltungen verfasst werden konnten.

Die Typen (I.) und (III.), zwischen denen durch Intertextualitätshinweise markierte Textbezüge bestehen können, ${ }^{21}$ lassen sich als Vorlesungsankündigungen $^{22}$ kategorisieren, insofern als sie in Bezug auf zukünftiges Verhalten orientierend wirken sollten, während durch (II.) bereits erfolgte Veranstaltungen retrospektiv erfasst wurden. Mitunter werden Vorlesungsankündigungen und Rechenschaftsberichte durch Ausblendung der Perspektive zu einer gemeinsamen Kategorie Vorlesungsverzeichnis (im weiteren Sinne) zusammengefasst (etwa bei Bruning 2011: 270). Obwohl eine solche Benennungsstrategie irreführend erscheint, ist ein passenderes Hyperonym für diese vorlesungsbezogenen Texte bislang nicht verfügbar, und das vermutlich aus gutem Grund. Die Vorlesungsankündigung leistet im Kern eine Einführung und ggf. Explikation der Textthematik von einer oder mehreren Vorlesung(en). Die Rechenschaftsberichte sind dagegen rekapitulierende Texte, bei denen im Prinzip ein Primärtext $\mathrm{T}$ in der Form eines Sekundärtextes t reproduziert wird (vgl. Kretzenbacher 1990: 11). Auch wenn beide an der Oberfläche eine reduzierte Repräsentation der jeweiligen Vorlesung bieten, geschieht dies doch auf völlig unterschiedliche Weise: im einen Fall durch die Expansion des Textthemas, im anderen Fall durch die Reduktion des Ausgangstexts (d.h. der gehaltenen Vorlesung).

Das nachfolgende Schema bildet die gängige quellentypologische Strukturierung dieses Überlieferungsfelds ab und hat v.a. heuristischen Wert. Eine

21 So verweist etwa Christian Thomasius als Professor in Halle regelmäßig vom Lektionskata$\log$ auf seine persönlichen Vorlesungseinladungen: z.B. 1694 „in peculiari programmate“ (Codex Lectionum fol. 4r), 1696 ,indicabit peculiaris schedula“ (fol. 6r), 1701 „peculiari Programmate in idiomate vernaculo conscripto“, „peculiari programmate Germanico“ (fol. 16r, 17r).

22 Bei Pozzo (1999: 73 Anm. 38) abweichend gebraucht für den einzelnen Eintrag als elementaren Baustein eines Vorlesungsverzeichnisses, bei Oberhausen \& Pozzo (1999: XI Anm. 1) für „die einzelnen Einträge aus den Catalogi Praelectionum, für die handschriftlichen Blätter der Dozenten am Schwarzen Brett und die Programmata“. 
exhaustive Analyse der verschiedenen vorlesungsbezogenen Texte müsste auch Vorlesungsmitschriften und -nachschriften berücksichtigen, für die historisch-textlinguistische Vorarbeiten ebenfalls weitgehend fehlen.

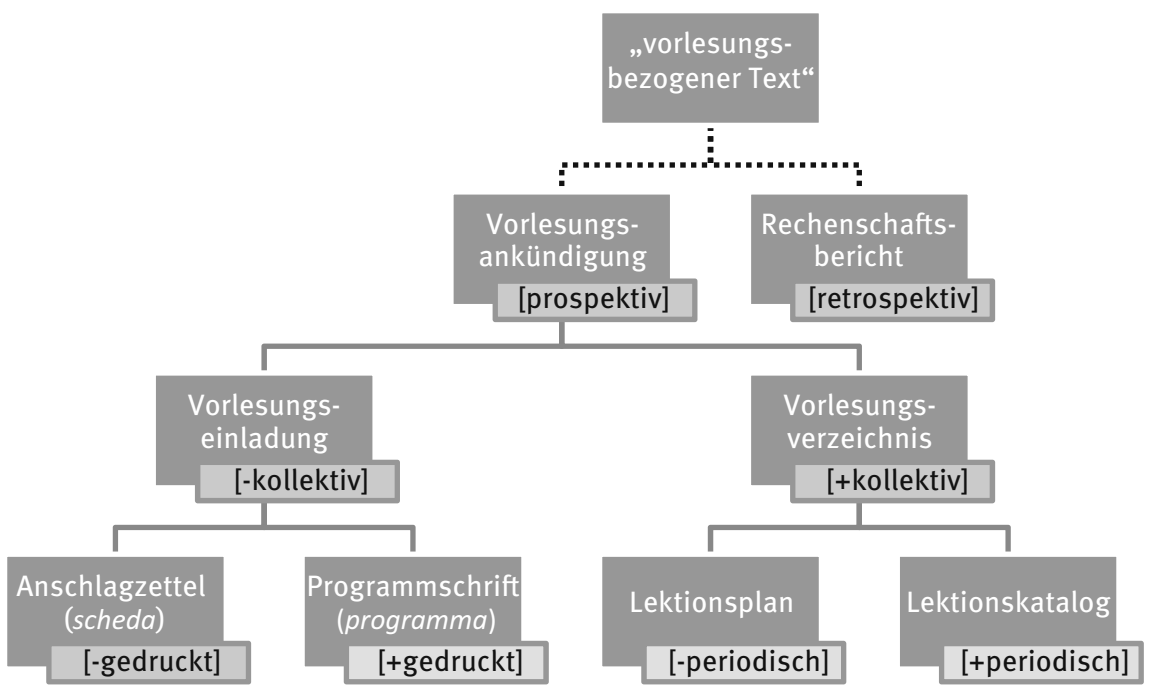

Inzwischen werden vorlesungsbezogene Texte mit Erfolg als wichtige Erkenntnisquelle für die Rekonstruktion des Fächer- und Themenspektrums bzw. des historischen Unterrichtsgeschehens herangezogen, wobei sie sich im Idealfall einer günstigen Überlieferungssituation wechselseitig ergänzen: Während die Lektionskataloge als das „Unterbewusstsein“ der akademischen Welt (Clark 2006: 67) einen wenig detaillierten, jedoch extensiven Überblick über das Unterrichtsangebot liefern, ergänzen die Programmata das inhaltliche und mitunter auch das didaktische Konzept für einzelne Vorlesungen; die Rechenschaftsberichte (und im Idealfall sogar noch studentische Kolleghefte) erlauben schließlich einen Abgleich mit der Unterrichtsrealität (vgl. Le Cam 2016: 107 u. 120 f.).

Allerdings ist selbst bei den Publica längst nicht immer garantiert, dass die Veranstaltungen am Ende überhaupt stattgefunden haben. Aus Leipzig z.B. berichtet Reichel (1742: 20):

Was die öffentlichen Lectiones betrifft, so läst man in den gedrukten Lections-Catalogo, große Menge herrl. Collegia, die man halten will, eindrucken [...] Kommt einer und spricht, weils im Catalogo Lectionum da stünde, so müste es ex promisso gelesen werden, so heist es, man müsse ja was hineinsetzen, weil der Catalogus nach Hofe geschickt würde. 
Tatsächlich kündigt der Leipziger Lektionskatalog von 1777 knapp 300 Veranstaltungen für ca. 800 Studenten an (vgl. Huttner 2007b: 198). Andernorts dürften die Verhältnisse ähnlich gewesen sein. In Salzburg etwa kommt Matthäus Fingerlos, der Regens des dortigen Priesterseminars, in einer anonym veröffentlichten Schmähschrift Ueber öffentliche Lehranstalten insbesondere über Lektionskataloge auf Universitäten (zur Autorschaft vgl. Marquart 1977: 95-97) zu dem vernichtenden Urteil, dass viele Vorlesungen nur angekündigt, nicht aber gehalten würden und dass der dortige Lektionskatalog ,eine der grösten gelehrten Windbeuteleyen sey“ (Fingerlos 1798: 206 u. 199).

Unabhängig von der Frage, ob angekündigte Vorlesungen am Ende gehalten wurden, sind die durch Lektionskataloge und Programmschriften annoncierten Lehrabsichten ein ,aufschlußreicher Indikator für das die akademische Lehre leitende Wissenschafts- und Disziplinverständnis“ (Huttner 2007a: 217), ein Indikator, an dem sich sowohl die einzeldisziplinäre als auch die allgemeine Wissenschaftsentwicklung zuverlässig ablesen lässt (vgl. dazu Blanke 1983: 205).

\section{Thomasius' Gracián-Programma von 1687}

In Leipzig wurden ab 1681 einmal jährlich, am Sonntag Rogate als dem offiziellen Beginn des akademischen Jahres, vom Rektor alle Vorlesungen für die gesamte Universität und das ganze Studienjahr über einen Plakatdruck im Folioformat bekannt gegeben, und zwar in der Regel neben den öffentlichen auch schon die honorarpflichtigen collegia privata (vgl. Huttner 2007a: 193 u. 199 f.). Allerdings galt dieser Usus zunächst nur für die wenigen ordentlichen Professoren (ordinarii), also solche, die Fakultätsstellen innehatten. Privatdozenten ${ }^{23}$ wie Christian Thomasius konnten ihren Unterricht erst ein knappes Jahrhundert später über die offiziellen Lektionskataloge bekannt machen. ${ }^{24}$ Das Gros der „extraordinären“ Universitätslehre (dazu Müller 2001: $191 \mathrm{f}$.), die z. B. in Jena kurz nach 1700 nicht ganz 70\% der Lehre an der Philosophischen Fakul-

23 Zum Status des Privatdozenten (typischerweise ein doctor oder magister legens) im 18. Jh. vgl. etwa Tütken (2005: 357) und Rasche (2009b), zur Entstehung und Entwicklung der Privatvorlesung allgemein vgl. Horn (1897) und Huttner (2007b: 158-163).

24 In Leipzig ab dem Sommersemester 1773 (vgl. Huttner 2007a: 209f.); vergleichbare Entwicklung an anderen Universitäten: in Jena zum Wintersemester 1768/1769 (vgl. Rasche 2008: 33), an allen preußischen Universitäten ab dem Wintersemester 1770/1771 im offiziellen Katalog (vgl. Pozzo 1999: 73), in Königsberger Zeitungen allerdings bereits in den Sechzigerjahren (vgl. Oberhausen \& Pozzo 1999: XIV u. XLII f.). 
tät ausgemacht haben dürfte (vgl. Rasche 2008: 34), entzieht sich weitgehend unserer Kenntnis, sofern nicht die betreffenden Personen auf eigene Kosten ${ }^{25}$ akademische Programmschriften drucken ließen, um die Leipziger universitäre Öffentlichkeit über die geplanten Kollegs zu informieren.

Eine solche Programmschrift veröffentlichte Christian Thomasius im Jahr 1687 als damals noch relativ unbekannter ${ }^{26}$ Leipziger Jurist - er war zuvor in Gelehrtenkreisen am ehesten durch zwei Bigamie-Dissertationen aufgefallen, in denen er u. a. eine naturrechtliche Gleichrangigkeit von Polygynie und Polyandrie konstatierte. ${ }^{27}$ Das undatierte Programma von 1687 erschien bei Moritz Georg Weidmann in Leipzig unter folgendem Titel:

Christian Thomas eröffnet Der Studirenden Jugend zu Leipzig in einem DiscouRs Welcher Gestalt man denen Frantzosen in gemeinem Leben und Wandel nachahmen solle? ein COLLEGIUM über des GRATIANS Grund-Reguln/ Vernünfftig/ klug und artig zu leben. ${ }^{28}$

Der Ausdruck Discours ist hier in der ursprünglichen, seit dem 16. Jahrhundert bezeugten Bedeutung ,(wissenschaftliche) Abhandlung' gebraucht. ${ }^{29}$ Vom Titelblatt der Programmschrift wird also zugleich eine Abhandlung zu der zitierten Themenfrage und eine Vorlesung ${ }^{30}$ angekündigt. Tatsächlich bewarb das Heft sogar zwei Collegia: Thomasius stellt darin in Aussicht, die theoretische Sittenlehre nach seinen eigenen Institutiones Jurisprudentiae divinae (IJD) zu unterrichten, die Textgrundlage der praktischen Sittenlehre sei das sog. HandOrakel (Oráculo manual y arte de prudencia), die aus 300 Maximen („Grund-

25 Nach Pozzo (2012: IX) erfolgte die Distribution der Texte „unbound free of charge at the expenses of the professor“.

26 In den KTS (56) räumt Thomasius ein, die Publikationstätigkeit dieser Jahre habe v. a. dazu gedient, sich aus der „obscurität“, in der er damals noch „verborgen“ gewesen sei, „hervor zu thun und bekant zu machen“.

27 EJH III (4): „Dieses entsinne ich mich wohl, daß etlichen unter ihnen [= den Mitarbeitern der Acta Eruditorum], die Disputation Anno 85. de crimine bigamiæ gar nicht anstund“. Allerdings reagierte auch Pufendorf skeptisch (vgl. BW Nr. 30). Zur Einordnung von Thomasius' Position vgl. etwa Doyé (2012: $101 \mathrm{f}$.).

28 Exemplare des 40-seitigen (38 S., [1] Bl.; 4º) Originalprogramms haben sich in Dresden, Erlangen-Nürnberg, Halle, München und Wolfenbüttel erhalten (s.u. VE 5), wobei die Bibliothekskataloge z. T. ungenaue Angaben zum Erscheinungsjahr machen („ca. 1700“ [BSB], „ca. 1690“ [SLUB], entsprechend „1690“ im DTA). Das Erscheinungsjahr ist jedoch u. a. durch die Angabe im Catalogus Scriptorum von 1693 (Nr. II/5: „Ann. 87.“) und das Nachwort zur Ausgabe in den Kleinen Teutschen Schrifften gesichert: „Dieses ist mein erstes Teutsches Programma, so ich in Leipzig Anno 87. verfertiget“ (KTS 53).

29 Zur Entlehnungsgeschichte von Discours vgl. Jones (1976: 293) und Kämper (1999; mit Thomasius-Belegen); zur Verwendung in der Frühaufklärung vgl. Wille (1991: 34 f.).

30 Zur Bedeutungsentwicklung von collegium, das im späten 17. Jh. bereits weitgehend synonym mit lectio ,Vorlesung` verwendet wurde, vgl. Horn (1897: 17-22). 
Reguln“) bestehende Klugheitslehre des spanischen Jesuiten Baltasar Gracián von 1647. Die Inhalte der beiden angekündigten Vorlesungen spielen im Discours allerdings nur eine untergeordnete Rolle. ${ }^{31}$ Dagegen nimmt die Frage der akademischen Sprachwahl breiten Raum ein, da es sich um Thomasius' erste volkssprachige Vorlesungseinladung ${ }^{32}$ handelte.

Die Textauswahl ist vor dem Hintergrund $\mathrm{zu}$ sehen, dass Vorlesungen im ausgehenden 17. Jahrhundert zunehmend der Exegese eines maßgeblichen Lehrbuchs dienten. Der von den Statuten vorgeschriebene Kanon autoritativer Texte wurde dabei mehr und mehr durch Kompendien ersetzt, die ,in möglichst geordneter und übersichtlicher Weise in ein bestimmtes Sachgebiet einführen sollten“ (Huttner 2007b: 163). Das Hand-Orakel war hierbei für Leipziger Studenten ein hochaktueller und attraktiver Text. Gerade eben war in Leipzig von dem Juristen Johann Leonhard Sauter die erste deutsche Übersetzung vorgelegt worden. ${ }^{33}$ Auch die französische Ausgabe von Nicolas Amelot de La Houssaie war erst drei Jahre vor dem Thomasius-Kolleg erschienen (Amelot 1684).

Insofern diente die Nichterwähnung der (vermutlich auf Latein gehaltenen) IJD-Vorlesung auf dem Titelblatt wohl einer Optimierung der Werbewirkung. ${ }^{34}$ Vor seinen Studenten musste der Universitätslehrer damals, wie HansJoachim Heerde und Ulrich Joost (2006: 13) etwas ungnädig bemerken, durch „eine Attraktion um deren Gunst buhlen, ihren Marotten und jugendlichen Torheiten nachgeben“. Die zunehmende Adressatenorientierung erscheint als ein wesentliches Merkmal des politisch-galanten Gelehrtenideals der Frühaufklärung (vgl. Fulda 2008: 288). Gerade die Konzentration auf ein populäres Thema und einen zeitgenössischen Autor, aber vor allem auch die Sprachwahl ${ }^{35}$ waren Stimuli zur Erregung von Aufmerksamkeit und damit absatzfördernde Maß-

31 Das zu behandelnde Themenspektrum wird am Ende des Discours' und im Nachwort der Ausgabe von 1701 lediglich mit einigen kurzen Leitfragen angedeutet (,wer der Gracian gewesen? Was er sonst geschrieben? Was von diesem Buch absonderlich zuhalten?“ usw.), nicht aber durch propositiones detailliert entwickelt (vgl. KTS $51 \mathrm{u}$. 53-56).

32 Es war jedoch keineswegs seine erste akademische Programmschrift, wie Pozzo (2012: XII) irrtümlich annimmt (s. u. die Aufstellung der Leipziger Programmata VE 1-9).

33 Bei Longo (2012: 3 Anm. 19) irrtümlich dem Drucker Adam Gottfried Kromayer zugeschrieben. Die Erstauflage erschien 1686 unter dem Titel L' Homme De Cour Oder Balthasar Gracians Vollkommener Staats- und Welt-Weise (VD 17 3:605450W). Eine zweite, überarbeitete Auflage ließ Sauter mit geändertem Untertitel bereits im Jahr darauf in zwei Ausgaben erscheinen: VD 17 3:307733Z (Mainz: Kromayer) und VD 17 23:327968Z (Frankfurt, Leipzig: Kromayer). Zu den duodezformatigen Ausgaben Sauters vgl. Forssmann (1977: 272-274).

34 Schneiders (1993: XI) sieht in der Anknüpfung an Gracián gar einen „modische[n] Werbetrick“; zur Frage der erstaunlichen „Werbewirksamkeit“ des spanischen Jesuiten in protestantischen Universitätsmilieus vgl. differenziert Achermann (2003: 8 f.).

35 Die beiden anderen Faktoren trafen auch auf das IJD-Kolleg zu: Eine Veranstaltung zum Naturrecht anhand eines für studentische Belange konzipierten (s. Anm. 50) und zur Herbst- 
nahmen für einen jungen Gelehrten, der den Lebensunterhalt für sich und seine Familie aus seiner privaten Lehr- und Publikationstätigkeit, „von Collegiis und Bücher-Schreiben“, ${ }^{36} \mathrm{zu}$ erwirtschaften hatte. „Such private classes had to interest the student as a consumer, whose demands they in part created“, wie William Clark (2006: 62) treffend feststellt. Darin liegt zugleich auch die besondere wissenschaftsgeschichtliche Bedeutung der Privatlehre begründet sie führte zu einer „Ökonomisierung des Lehrbetriebs“ (Rasche 2008: 29) und war zugleich ein Einfallstor für wissenschaftliche Innovation, durch das zahlreiche neue Themen und Methoden an die frühneuzeitlichen Universitäten gelangten. Die collegia privata bereicherten die häufig in einem traditionellen Lehrkursus erstarrte Doktrin und liefen den Publica im 18. Jahrhundert zunehmend den Rang ab (vgl. Bruning 2011: 286; Le Cam 2016: 109).

Die genauen Abläufe bei Leipziger Vorlesungsankündigungen dieser Zeit lassen sich anhand der gut dokumentierten Lectiones privatae Anti-Atheisticae, die der Theologe August Pfeiffer im Sommer 1689 abhielt, anschaulich nachvollziehen. ${ }^{37}$ Pfeiffer hatte für seine Vorlesung eine gegen Thomasius gerichtete Programmschrift verfasst, die auf den 7. April („Dom. Quasim.“) datiert war und an diesem Tag, einen Monat vor Vorlesungsbeginn, öffentlich angeschlagen werden sollte (EJH III: 64 u. 80). Die Datumsangabe des Programms entsprach hier also dem Zeitpunkt der geplanten universitätsöffentlichen Bekanntgabe und nicht dem Datum der Manuskriptfertigstellung oder des Imprimaturs. Vor dem angegebenen Zeitpunkt sind somit noch die Druckfreigabe durch die Fakultät und der Druckvorgang selbst anzusetzen. Pfeiffer weist darauf hin, dass er „Censuram Decani suæ Facultatis gebührend requiriret“ habe (EJH III: 66), was die Theologische Fakultät ihm in einer Stellungnahme auch bereitwillig bestätigt. So habe

Herr D. Aug. Pfeiffer Hebr. L. P. P. ein Collegium Privatum Anti-Atheisticum auf morgenden Sontag Quasimodogeniti zu intimiren sich bey mir jetziger Zeit Decano gewöhnlicher massen angemeldet, das Programma und Quæstiones Anti Atheisticas, darüber er in solchem Collegio zu lesen gemeynet, überreichet, und die Censuram und subscriptionem erhalten. (EJH III: 67)

messe 1687 gerade erst erscheinenden Lehrbuchs (BW 32 Anm. 2) dürfte für Studenten durchaus attraktiv gewesen sein. Ein wesentlicher Unterschiede zwischen beiden Vorlesungen war jedoch, dass das Programm in Bezug auf das Gracián-Kolleg die Erwartung auf einen Unterricht in der Muttersprache weckte.

36 Summ. Anzeige (246); vgl. auch VGH II (69). Noch in EJH III (4) hält er fest: Um damals die Familie ernähren zu können, sei der Tag „mit vielen Stunden Collegiorum privatorum \& privatissimorum besetzet“ gewesen.

37 Die Pfeiffer-Vorlesung begann laut Thomasius nach der Ostermesse (EJH III: 64), also in der Woche vom 6. Mai. An anderer Stelle macht er abweichende, vermutlich aber nur unge- 
Sobald Zensur und Druck erfolgt waren, zirkulierten die Programmschriften, sodass Thomasius bereits vier Tage vor der geplanten Veröffentlichung ein Exemplar des Pfeifferschen Texts zugespielt werden konnte (EJH III: 64). Allerdings finden sich auch andere Datierungsstrategien. So teilt Thomasius im Zusammenhang mit einer möglichen Rückberufung nach Leipzig am 17. April 1705 dem Dresdner Kabinettssekretär Georg Ernst Pfingst mit:

Ich habe indeßen, $u$. weil ich wohl vorhergesehen, daß die sache sich nicht so geschwind poußiren [=,voranbringen'] laße, ein programma drücken laßen, welches morgen fertig wird, und mit ehester gelegenheit überschicket werden soll, was ich diesen Sommer allhier zu lesen gesonnen binn. (HStA Dresden, 10026 Geheimes Kabinett Loc. 00554/021, fol. 4/5r)

Diese Einschätzung war aber wohl zu optimistisch, da Pfingst am 19. April zunächst nur den ersten Bogen erhielt:

Ich übersende hiebey den ersten Bogen von demjenigen, was der H. Thomasius künftig $\mathrm{zu}$ lesen und zu schreiben gesonnen, so bald der überrest fertig, folget solcher gleichfals. (HStA Dresden, 10026 Geheimes Kabinett Loc. 00554/021, fol. 7v)

Ohnehin hatte Thomasius das Programma bereits auf den 15. April datiert (vgl. Lieberwirth 1955: Nr. 176). Die Texte wurden also erst einige Tage nach dem aufgedruckten Publikationsdatum verfügbar.

In der Regel beanspruchten Zensur und Druck einer akademischen Programmschrift wohl nur geringe Zeit. Das Manuskript zu seinem ersten Programma (VE 1) hat Thomasius vermutlich im August 1683 an Jacob Born (d. Ä.) gesendet, der als Ordinarius der Juristenfakultät für die Zensur juristischer Programmschriften zuständig war (vgl. Zedler Bd. 25: Sp. 1793). Born regte zunächst in einem Schreiben vom 28. August gewisse Änderungen an und erteilte am 4. September schließlich das Gut zum Druck des überarbeiteten Texts. ${ }^{38}$ Dieser konnte damit bereits fünf Tage später veröffentlicht werden. Eine echte Hürde wurde die Zensur für Thomasius allerdings, als Born sich 1688 von der Theologischen Fakultät überzeugen ließ, dass das Naturrecht „mehr für die Philosophische oder auch wohl Theologische Facultät, als für die Juristische“ (VGH II: 271f.) ein Thema sei. Da er seine Programmschriften in der Folge in Leipzig nicht mehr zensiert bekam, musste er mit deren Druck ab VE 7 nach Halle ausweichen. ${ }^{39}$

naue Angaben: Seine eigene Vorlesung habe am 10. Juni begonnen, Pfeiffer habe sein „Collegium etwan acht Tage vorher“ (101) angefangen.

38 Hamburg, Staats- und Universitätsbibliothek, Sup. ep. 51, Bl. 7 und 9. Gedruckt bei: BW Nr. 14 u. 18.

39 So sei 1688 ,besagtes mein Buch [= IJD], so viel librum I. anlanget, allhier zu Leipzig von dem Herrn Ordinario Facultatis Juridicae, die übrigen beyden Bücher aber zu Halle von einem 


\section{Thomasius' Vorlesungseinladungen bis 1690}

Als Datum für die Gracián-Vorlesung ist der 31. Oktober alten Stils ${ }^{40}$ gängig geworden, ein Datum, welches die passende symbolische Aufladung bereits mitliefert: Thomasius' „Luthertat“ fiele damit ausgerechnet auf den Reformationstag. Diese Berechnung des ehemaligen Rostocker Universitätsbibliothekars Adolph Hofmeister aus dem Jahr 1887 fand rasch Eingang in die Handbücher und gilt heute allgemein als kanonisches Wissen. Allerdings wird dieses Datum in der Literatur z.T. als Zeitpunkt der Unterrichtsankündigung, z. T. als Zeitpunkt des Unterrichtsbeginns in Anspruch genommen. Falsch ist beides, da Hofmeisters Berechnung auf einem Lesefehler beruht. ${ }^{41}$ Insofern muss der genaue Zeitpunkt der Ankündigung ebenso wie der des Unterrichtsbeginns als unklar gelten.

Gegen Ende des Discours' werden die beiden Veranstaltungen zur Moralphilosophie ${ }^{42}$ mit folgenden Worten bekannt gegeben:

IJD-Vorlesung (theoretische Sittenlehre): „Was l' honnêtete anlanget/ bin ich gesonnen/ die Maximen des Göttlichen Rechts [...] nach Anleitung meiner Institutionum Jurisprudentiæ divinæ, wo GOtt will/ auff dem Montag nach der Zahlwoche nach mittags nach zwey Uhr wiederum zuerklären anzufangen/ und binnen dato und Ostern künfftiges Jahres zu vollenden“ (VE 5: 37).

Gracián-Vorlesung (praktische Sittenlehre): Thomasius räumt ein, er sei in Bezug auf die „grundgesetze d' un bel esprit, du bon gout et d' un galand homme“ selbst noch Lehrling und müsse auf das Buch Graciáns zurückgreifen, welches er „zwischen hier und Ostern“ erklären werde, wobei er „auff erwehnten Montag nach der Zahlwoche vor mittag ümb 9. Uhr den Anfang zu machen“ gedenke (VE 5: $37 \mathrm{f}$.).

Im Frühjahr wäre der besagte Termin tatsächlich identisch gewesen mit dem Montag nach Rogate als dem offiziellen Beginn des akademischen Jahres. Die Gracián-Vorlesung hätte demnach am 2. Mai (alten Stils) um 9 Uhr morgens

darzu bestellten Lutherischen JCto und Consistorial Rath censiret und gedruckt worden“ (EJH III: 155).

40 Der 10. November nach neuem Stil, welcher in Sachsen erst 1700 eingeführt wurde.

41 Hofmeister präzisierte mit seiner Miszelle anonyme Berechnungen im selben Zeitschriftenband (Die Grenzboten 46, 2. Vierteljahr, 545 f. [„Ende September oder Anfang Oktober“] u. 599 [,24. Oktober“]), übernahm von diesen jedoch einen falschen Zeitpunkt für den Messebeginn. Diese habe angeblich nach den Angaben im Leipziger Haupt- und Geschicht-Calender des Jahres („3. sont. nach michael“) am dritten Sonntag nach dem Michaelisfest angefangen. In Wahrheit hat die Ordinalzahl im Kalender allerdings Gliederungsfunktion und dient lediglich der Nummerierung der drei Leipziger Messen: 1. Neujahrsmesse, 2. Ostermesse, 3. Michaelismesse (vgl. Kalender C).

42 Zu Thomasius' Einstieg in die Sittenlehre vgl. Steinberg (2005: 49-51). 
begonnen und wäre um Ostern des Folgejahres abgeschlossen gewesen. ${ }^{43}$ Allerdings bemerkt Thomasius im Discours, er beabsichtige, „diesen Winter durch/ denen so dießfalls meine Lehrart anstehet/ anleitung zugeben“ (VE 5: 36). Die Vorlesung fand also eindeutig im Wintersemester statt, wenngleich etwas früher als bislang stets angenommen. Um 1700 wurde die Herbstmesse nämlich noch immer stiftungsgemäß $\aleph^{44}$ am Sonntag nach Michaelis durch das mittägliche Einläuten eröffnet, mit dem Ausläuten am darauffolgenden Sonntag begann dann die sog. Zahlwoche. Da das Michaelisfest (29.9.) im fraglichen Jahr auf einen Donnerstag fiel, begann die Messwoche am Sonntag, dem 2. Oktober 1687, die Zahlwoche dauerte vom 9.-16. Der von Thomasius angegebene Montag nach der Zahlwoche war somit der 17. Oktober. Die berühmte Vorlesung wurde also nicht am Reformationstag eröffnet, sondern am Festtag des Florentius, eines Trierer Bischofs aus dem 3. Jahrhundert, ${ }^{45}$ was das Heroisierungspotential empfindlich schmälert. Dass dieses Datum korrekt ist, belegt jedenfalls eine Disputation des Folgejahrs, bei der Thomasius nachweislich denselben Berechnungsmodus verwendet hat. ${ }^{46}$

Schwieriger $\mathrm{zu}$ beantworten ist die Frage nach dem thomasianischen Thesenanschlag, ${ }^{47}$ dem Zeitpunkt der volkssprachigen Vorlesungsankündigung als dem eigentlichen Skandalon. Mehr als drei Jahrzehnte nach den Vorgängen meint Thomasius sich zu erinnern, dass diese „gegen die Oster-Messe“ erfolgt sei (EJH III: 4) - ein Hinweis, der bislang meist ignoriert wurde, ${ }^{48}$ auf den ersten Blick aber durchaus glaubwürdig erscheint. Die Leipziger Lektionskataloge wurden wie erwähnt am Sonntag Rogate als dem offiziellen Beginn des akademischen Jahres vom Rektor für das gesamte Studienjahr publiziert. ${ }^{49} \mathrm{Im}$ fraglichen Jahr war dies der 1. Mai, zwei Wochen zuvor hatte die Leipziger Frühjahrsmesse (Oster- oder Jubilatemesse) begonnen. Da im ausgehenden

43 Eine solche Laufzeit wäre zu dieser Zeit in Leipzig nicht ungewöhnlich gewesen (vgl. Huttner 2007a: 205).

44 Zur Berechnung der jährlichen Messetermine vgl. etwa den Kalender E (s.v. Leipziger messe), Döring (1837: 187) und Hasse (1885: 173-177 u. 188).

45 Die zeitgenössischen Leipziger Schreibkalender machen hier jedoch widersprüchliche Angaben: So hat der Kalender D auf das Jahr 1700 für den 17. 10. „Florent.“, was in den Kalendern A/B für 1658 und 1679 zu Florentinus aufgelöst wird (statt Florentius). Der Kalender C für 1681 hat dagegen - wohl irrtümlich - „Florentina“; zu Florentius vgl. Grotefend (1891-1898: 102).

46 In VE 7 legt er die erste Disputation des WS 1688 auf „künfftigen Sonnabend nach der Zahlwoche/ wird seyn der 20. October“ (KTS 215).

47 Zur Pragmatik der akademischen schwarzen Bretter und zur Praxis des Anschlagens vgl. Prinz (2019).

48 Vgl. aber Pörksen (1986: 45; 1990: 21).

49 So bestätigt z. B. Rotth (1692: 21) für das darauffolgende Jahr, dass „der Herr Rector Dom. Rogate, Anno 1688. die Lectiones Professorum“ im Lektionskatalog angekündigt habe. 
17. Jahrhundert zwar Ordinarien ihre honorarpflichtigen Privatkollegien in den Lektionskatalogen ankündigen konnten, nicht jedoch Privatdozenten (s.o.), muss dieses Datum allerdings nicht zwingend auch für sein Programma gegolten haben.

Zweifelhaft erscheint zudem Thomasius' Behauptung, er habe nach der Publikation des Gracián-Programms „,zugleich über die hernach edirte institutiones Jurisprudentiae divinae und introductionem ad Philosophiam Aulicam zu lesen“ angefangen (EJH III: 4). Die beiden Kollegs fanden jedoch in unterschiedlichen Semestern statt: Über die Philosophia Aulica las Thomasius erstmals im Sommersemester 1687 (s. VE 4), das IJD-Kolleg fand gleichzeitig mit dem Gracián-Kolleg im Wintersemester statt (s. VE 5). Zuvor hatte er im Rahmen seines ersten juristischen Vorlesungszyklus bereits im Sommer- und Wintersemester 1686 über das Naturrecht gelesen und dazu eine frühe Fassung der IJD herangezogen: ${ }^{50}$

- $\quad$ SS 1686 \& WS 1686/1687: Naturrecht nach den Proto-IJD (s. VE 3)

- SS 1687: Philosophia Aulica (s. VE 4)

- $\quad$ WS 1687/1688: Theoretische Sittenlehre nach den IJD (s. VE 5)

50 Thomasius' zweites Pufendorf-Kolleg markiert den Beginn der Arbeit an den IJD (vgl. IJD, Proœm. §21). Die Vorlesung begann lt. VE 3 (= PT 33 f.) im Sommersemester 1686 als Teil eines zweijährigen Vorlesungszyklus. Auf Wunsch der Studenten werde er Pufendorfs Werk De officio hominis et civis allerdings nicht mehr „Discurs-weise“ vortragen, sondern „,in Form gewisser Lehrsätze zu Papier bringen“ (RG § 21). Er werde das Naturrecht im ersten Jahr jeweils Mo. und Di. von 18-19 Uhr lesen und dabei „Institutiones Juris Naturæ MSS. seorsim Auditoribus meis communicandas" vorstellen. Hierbei scheint es sich um eine erste handschriftliche Entwurfsfassung von Teilen der IJD gehandelt zu haben. Bereits im Herbst lagen dann auch gedruckte Passagen vor: Spener konnte im Nov./Dez. 1686 Teile der IJD lesen und kommentieren (BW Nr. 31 f.). Thomasius’ Anmerkungen vom 10.11. 1686 zu Speners Diskussionspunkten lassen dabei erkennen, dass dieser die beiden ersten Kapitel (von insgesamt vier) des 1. Buchs der IJD bekommen hatte, und zwar offenbar schon in der gedruckten Fassung. Die vereinzelt erwähnten Paragraphen- und Seitenangaben stimmen nämlich bereits zur späteren Druckausgabe (vgl. BW Nr. 32 Anm. 2). Das Feedback Speners wurde dann ins Proœmium aufgenommen („Ad. 1.“ des Briefs betrifft IJD I/1, § 29 und wird in Proœm. §38 thematisiert, „Ad. 14.“ zu IJD I/2, §125f. in Proœm. §49), der Text der IJD selbst aber nicht mehr grundlegend geändert. Die ersten Bögen mit den Kap. 1 und 2 wurden also wohl im Okt. 1686 gedruckt, möglicherweise um zum Vorlesungsbeginn des WS am 18. Okt. verfügbar zu sein. Eine Verwendung im Unterricht belegen auch Äußerungen des Verlegers Moritz Georg Weidmann, mit dem Thomasius Anfang des Jahres 1691 wegen der Begleichung wechselseitiger Auslagen im Streit lag. Am 11. 2. versuchte Weidmann, Thomasius' Honorarforderung für die IJD drastisch zu reduzieren. Er habe die IJD damals in einer Auflage von 1000 Exemplaren gedruckt, von der noch die Hälfte übrig sei. Thomasius habe eigtl. auf ein Honorar verzichtet, damit diese „damalß zulieb dero Collegiorum getruckt wurden“ (Lehmstedt 1992: 355). Am 14. 3. bekräftigt er, bei den IJD habe Thomasius ihn „zum Verlag genöthiget, vnd damals kein gl. darfür begehret, dann es zu dero nuzen vor dero Colleg: trucken laßen“ (ebd. 358). Ein etwas rätselhafter Hinweis findet 
Thomasius' Angaben zur Publikation des Gracián-Programms passen somit nicht zusammen. Zwar beruht die Dokumentation der verschiedenen Händel (EJH, VGH) in der Regel auf einer beeindruckend akribischen Auswertung umfangreicher Quellensammlungen, sodass Thomasius noch Jahrzehnte später wörtlich aus Briefen zitieren und ganze Vorlesungskonzepte wiedergeben kann. Die konsequente Archivierung der entsprechenden Unterlagen scheint bei dem notorisch unwilligen Briefschreiber Thomasius (dazu BW S. XV) aber eine Reaktion auf die ab 1688 sich zuspitzenden juristisch relevanten Konflikte gewesen zu sein. Für die Zeit vorher bleibt die Darstellung vergleichsweise unscharf.

Entweder hat Thomasius also bereits Mitte April das Kolleg im Voraus für das Wintersemester bekannt gegeben oder seine spätere Erinnerung ist hinsichtlich des Ankündigungstermins unzuverlässig. Um dies zu klären, ist es notwendig, einen Blick auf die bislang nicht untersuchte Datierungspraxis seiner Vorlesungseinladungen ${ }^{51}$ (=VE) zu werfen. Dafür wurden alle zu ermittelnden lateinischen wie deutschen Programmschriften und Anschlagzettel bis 1690 ausgewertet.

\section{Zur Darstellung}

Universitätsort und Semesterangabe beziehen sich jeweils auf das Studienhalbjahr, in dem die angekündigten Veranstaltungen begannen. Darauf folgt zunächst der kürzere Werktitel aus PL und KTS; die ausführlichen Originaltitel sind separat vermerkt. ${ }^{52}$ Von den bekannten Textausga-

sich in Weidmanns großer Buchrechnung, die gegen Ende einen undatierten Eintrag zu den IJD enthält. Demnach habe Thomasius neun Exemplare von „Jus Divin: $4^{\circ}$ Schreibepapp.“ erhalten, außerdem weitere 21 oktavformatige Exemplare, davon vier auf „Schreibepapp.“, 17 auf „Druck Pappier“ (ebd. 342). Die auf 1688 datierte Druckfassung der IJD erschien in Oktav; von einer Quartausgabe ist hingegen nichts bekannt. Möglicherweise hat man es hier also mit dem im Oktober 1686 erschienenen ersten Faszikel der IJD zu tun.

51 Mit den fraglichen Texten wird allerdings nicht nur zu Vorlesungen i. e. S. eingeladen, sondern häufig auch zu den sonstigen Lehrveranstaltungstypen, die im späten 17. Jh. im Rahmen der akademischen Privatlehre angeboten wurden, etwa zu collegia disputatoria, also privaten Disputationsübungen (dazu etwa Marti 2011: 301). VE 1 und VE 2 kündigen sogar ausschließlich ein solches Disputatorium an. Da diese Angebote jedoch als ein Subtyp der praktischen Vorlesung angesehen wurden (vgl. Zedler s. v. Vorlesung ${ }_{2}$ ), spricht nichts gegen eine Verwendung des Terminus Vorlesungseinladung.

52 Zwar sind die verschiedenen Überlieferungen in der Regel textidentisch, die Titel können jedoch markant abweichen. Dass z. B. VE 11 nur die Lectiones publicas betraf, geht aus dem Originaltitelblatt, nicht aber aus der Überschrift in PL hervor. 
be n werden zuerst die auffindbaren Originaldrucke aufgelistet (=a.), dann in chronologischer Reihenfolge die Neuauflagen und Neuausgaben (= b., c. usw.). Nicht in allen Fällen haben sich allerdings Originale der Vorlesungseinladungen erhalten. Universitätsbibliotheken und -archive hatten wohl in der Regel keinen systematischen Sammelauftrag für solche akademischen Kleinschriften. So war beispielsweise bereits um 1720 die Programmschrift zu August Pfeiffers Lectiones privatae Anti-Atheisticae (1689) „in Buchläden wohl schwerlich zu bekommen, auch in vielen Bibliothequen rar“ (EJH III: $69 \mathrm{f}$.).

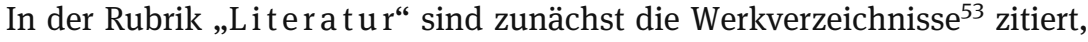
dann eine Auswahl wichtiger Titel zum jeweiligen Text. Die Spra changabe bezieht sich auf die Vorlesungseinladung, nicht auf die angekündigten Kollegs, deren Unterrichtssprache oft unbekannt ist. Im Kommentar ist bei wörtlichen Zitaten jeweils auf die Textfassung von PT (1724) bzw. KTS (1701) verwiesen. Datumsangaben erfolgen nicht doppelt, sondern nach dem julianischen Kalender, der in den betroffenen protestantischen Territorien bis 1700 in Gebrauch war. Bei späteren Berichten aus der Zeit nach Übernahme des neuen Stils wird davon ausgegangen, dass Thomasius Datumsangaben vor 1700 nicht rückwirkend „gregorianisiert“ hat. ${ }^{54}$

\section{VE 1: Programma invitatorium ad audiendas disputationes ad partem I. Positionum Juris contractarum Ulrici Huberi. Lipsiæ 1683 - Leipzig, Wintersemester 1683/1684}

Originaltitel: „Permittente Magnifico JCtorum Ordine in Academia Lipsiensi ad ventilationem publicam Partis I. Positionum contractarum Celeberrimi JCti Ulrici Huberi \&c. Christianus Thomasius, Phil. \& J.U.D. cupidam Legum Juventutem officiosè invitat. “55

Ausgaben: <a.> -; <b.> Positiones sive Lectiones I (1685), s. pag. (auf die Leservorrede folgend); <c.> PT (1724: Nr. I, S. 1-13); <d.> PT (2010, Nr. I).

Literatur: Cat. II/3; Becker (1931: Nr. A/2); Lieberwirth (1955: 8) - Steinberg (2005: 11, 53, 201 Nr. 3); Steinberg (2010, viii).

Sprache: lat.

53 Von den Altverzeichnissen (Catalogus scriptorum) ist lediglich die erste Auflage (Halle 1693) aufgenommen.

54 So hat er beispielsweise auch seinen eigenen Geburtstag nie umgerechnet (vgl. Zenne 1954: 568).

55 Der Titel nach dem von Thomasius 1685 herausgegebenen ersten Band der Positiones sive Lectiones entspricht wohl dem Originaltitel, wie die vergleichbare VE 2 nahelegt, zu der sich das Orig. erhalten hat. 
Mit seinem ältesten bekannten Programm gibt Thomasius am 9. Sept. 1683, also gut einen Monat vor Beginn des Wintersemesters (am 15. Oktober), ein privatrechtliches „exercitium disputatorium“ (12) zum ersten Teil der Positiones sive Lectiones Juris Contractæ des friesischen Juristen Ulrik Huber bekannt. Diese Disputationsübung werde samstags zwischen 1 und 4 Uhr stattfinden, und zwar mit Genehmigung der Juristenfakultät in deren Hörsaal. Da keine Datumsangabe für den Beginn der Übung gemacht wird, dürfte sich Thomasius - wie stets auch sonst im Wintersemester (vgl. VE 5, 7, 9 u. 12) - am offiziellen Semesterbeginn orientiert haben. Die erste Übung hätte dann am 20. Oktober stattgefunden, 41 Tage nach der Einladung. Auch das im WS 1688/1689 angekündigte Disputatorium begann am Samstag nach der Zahlwoche, also in der Woche des allgemeinen Vorlesungsbeginns.

\section{VE 2: Programma simile ad Partem II. Positionum Huberi. 1684 - Leipzig, Sommersemester ${ }^{56} 1684$}

Originaltitel: „Permittente Magnifico JCtorum Ordine in Academia Lipsiensi ad ventilationem publicam Partis II. Positionum contractarum Celeberrimi JCti Ulrici Huberi \&c. Christianus Thomasius, Phil. \& J. U. D. Cupidam Legum Juventutem officiosè invitat. Lipsiæ, Typis Christiani GözI.“

Ausgaben: <a.> VD 17 14:060308D ([4] Bl., 4): $\mathrm{a}_{1}=$ SLUB Dresden, Diss.jur.civ. 310,34; <b.> Positiones sive Lectiones II (1684), s. pag. (nach Titelblatt); <c.> PT (1724: Nr. II, S. 13-28); <d.> PT (2010: Nr. II).

Literatur: Cat. II/4; Becker (1931: Nr. A/3); Lieberwirth (1955: 14) - Steinberg (2005: 11, 53, 201 Nr. 3); Steinberg (2010: ix).

Sprache: lat.

Mitten im laufenden Semester, am 27. Juli 1684 („Dom. IX. post Trin.“, 28), annonciert Thomasius die Fortsetzung der mittlerweile abgeschlossenen Disputierübung vom vorherigen Wintersemester (26), und zwar für den folgenden Samstag, also den 2. August. Die Einladung erfolgte somit sechs Tage vor Veranstaltungsbeginn.

56 Steinberg (2005: 11, 53 u. 202; 2010: ix) bucht die Veranstaltung für das Wintersemester. Sie begann allerdings zwei Monate vor Ende des Sommersemesters und setzte VE 1, die Übung des vorhergehenden Wintersemesters, fort. 
VE 3: Programma de lectionibus privatis super fundamentis Juris universi. 1686 - Leipzig, Sommersemester 1686

Originaltitel: unbekannt ${ }^{57}$

Ausgaben: <a. > -; <b.> PT (1724: Nr. III, S. 28-35); <c. > PT (2010: Nr. III). Literatur: Cat. II/1; Becker (1931: Nr. A/5); Lieberwirth (1955: 21) - Steinberg (2005: 11, 202 Nr. 5-8); Steinberg (2010: ix-x).

Sprache: lat.

Am Pfingstmontag, dem 24. Mai 1686, zwei Wochen nach dem offiziellen Semesterbeginn, kündigt Thomasius einen auf zwei Jahre angelegten Zyklus von juristischen Privatvorlesungen an, der vom Sommersemester 1686 bis zum Wintersemester 1687/1688 dauern werde und in dem zunächst das Naturrecht und das römisch-deutsche Privatrecht, später dann Lehnrecht, öffentliches und Kirchenrecht behandelt würden (PT 32). Die Vorlesungsreihe ziele darauf ab, „fundamenta Juris universi continuo discursu explicare“ (PT 33). Sie markiert für Thomasius den Beginn langjähriger Bemühungen um eine Reform der Juristenausbildung, zudem den Einstieg in die Arbeit an den IJD (dazu Anm. 50). Da die erste Sitzung bereits am folgenden Donnerstag stattfinden sollte, betrug die Ankündigungsfrist lediglich drei Tage.

\section{VE 4: Programma occasione lectionum privatarum de prudentia cogitandi \& ratiocinnandi. $1687^{58}$ - Leipzig, Sommersemester 1687}

Originaltitel: „B. C. D. Christianus Thomasius Studiosæ Juventuti In Academia Lipsiensi Lectiones Privatas de Prudentia Cogitandi \& Ratiocinandi intimat.“ Ausgaben: <a.> VD 17 29:721375H ([2] Bl., $2^{\circ}$ ): $\mathrm{a}_{1}$ = UB Erlangen-Nürnberg, H62/ AUA (261-VI)-57f; <b.> PT (1724: Nr. IV, S. 35-42); <c.> PT (2010: Nr. IV). Literatur: Cat. II/2; Becker (1931: Nr. A/6); Lieberwirth (1955: 23) - Steinberg (2005: 12, 46 Anm.171, 202 Nr. 9); Steinberg (2010: xi).

Sprache: lat.

Thomasius gibt am 1. Mai, dem Sonntag Rogate als dem offiziellen Ankündigungstermin für das neue Studienjahr, bekannt, er werde eine Logik-Vorlesung

57 Angesichts der Parallelität von Cat. II/1 („Intimatio Lectionum privatarum super fundamentis juris universi“) mit II/2 dürfte der Originaltitel wohl analog zu VE 4 gestaltet gewesen sein.

58 Mit VE 4 identisch ist auch die bei Luden (1805: 88) fälschlich für das Jahr 1689 zitierte „Intimatio lection. priv. de prudentia cogitandi et ratiocinandi“. Dabei kann sich nicht um eine spätere Programmschrift handeln, da die Intimatio lt. Luden vor der Introductio ad Philosophiam aulicam (1688) erschienen sei. 
„per semestre æstivum intra privatos parietes disserere“ (38). Tatsächlich war die Vorlesung Thomasius' erste Veranstaltung zu seiner 1688 publizierten Introductio ad Philosophiam aulicam (vgl. KTS 212). Die Programmschrift lässt bereits deren Grundzüge erkennen und wird später in VE 6 (Von den Mängeln der aristotelischen Ethik) in einem Exkurs auch auf Deutsch präsentiert (KTS 7579). Die erste Zusammenkunft für das Logik-Kolleg wird bereits für den übernächsten Tag angesetzt (,proximo Martis die“, 41).

\section{VE 5: Discours welcher Gestalt man denen Frantzosen in gemeinem Leben und Wandel nachahmen solle - Leipzig, Wintersemester 1687/1688}

Originaltitel: „Christian Thomas eröffnet der Studirenden Jugend zu Leipzig in einem Discours Welcher Gestalt man denen Frantzosen in gemeinem Leben und Wandel nachahmen solle? ein Collegium über des Gratians Grund-Reguln/ Vernünfftig/ klug und artig zu leben. zufinden bey Moritz George Weidemannen.“

Ausgaben: <a.> VD 17 14:002031T (38 S., [1] Bl.. 4): $\mathrm{a}_{1}=$ SLUB Dresden, Hist.univ.B.232,misc.27; $\mathrm{a}_{2}=$ SLUB Dresden, Phil.C.230,16; $\mathrm{a}_{3}=$ UB ErlangenNürnberg, H00/4 PHL-VIIII 56; $\mathrm{a}_{4}=$ ULB Halle, 01 A 6645 (23); $\mathrm{a}_{5}=$ BSB München, 4 Diss. 3764,33; $\mathrm{a}_{6}=$ HAB Wolfenbüttel, Xb 10246; <b.> KTS (1701, ${ }^{21707,}$ ${ }^{3} 1721$ : Nr. I); <c.> Weißbach (1711, ${ }^{2} 1715$ : s. pag., „Judicium“59 ); <d.> Sauer (1894); <e.> v. Opel (1894: 70-122); <f.> v. Düffel (1970: 3-49); <g.> KTS (1994: Nr. 1); <h.> Longo (2012: 15-42). <i.> DTA: urn:nbn:de:kobv:b4-200905198084. Literatur: Cat. II/5; Becker (1931: Nr. A/7); Lieberwirth (1955: 25) - KTS (1994: VI f.); Steinberg (2005: 12, 49 f., 202 Nr. 10 f.); Longo (2012: 3-13).

Sprache: dt.

Wenngleich aus werbetaktischen Gründen allein das „Collegium über des Gratians Grund-Reguln“ (also Baltasar Graciáns Hand-Orakel) im Titel des Discours' aufscheint, lädt Thomasius mit dem Programma zu zwei Vorlesungen zur Moralphilosophie (theoretische und praktische Sittenlehre) ein, die beide am 17. Oktober beginnen und bis Ostern 1688 dauern sollten (s. o.). Über Anlage und Inhalt der Gracián-Vorlesung ist fast nichts bekannt, da Thomasius sie später nicht veröffentlicht hat - wohl auch, weil er sich damals in der Materie noch als „Lehrling“ mit einer ,geringen Wissenschaft und Erfahrung“ sah (50). Zudem wurde das Gracián-Kolleg später nie wieder von ihm angeboten. Immerhin sind einige Leitfragen zur Vorlesung am Ende des Discours' und im Nach-

59 Zur Aufnahme des Discours' in die Gracián-Ausgaben von Selintes/Weißbach vgl. Forssmann (1977: 274 f.). 
wort der Ausgabe von 1701 skizziert (s. Anm. 31). Zwar wird die Unterrichtssprache für die beiden Kollegs nicht explizit in der Programmschrift vermerkt, die umfangreichen Einlassungen im Discours zur Frage des Gelehrtenlateins lassen jedoch ebenso wie die intensive Benutzung der deutschen Übersetzung des Hand-Orakels während der Vorlesung ${ }^{60}$ eigentlich nur den Schluss zu, dass zumindest das Gracián-Kolleg auf Deutsch gehalten wurde. ${ }^{61}$ Für die Nachmittagsvorlesung, die auf den lateinischen IJD beruhte, muss das jedoch nicht zwangsläufig gegolten haben. Leider lassen sich weder für diese, noch für spätere IJD-Vorlesungen (WS 1691/1692, SS 1700, SS 1711) ${ }^{62}$ eindeutige Hinweise auf die jeweilige Unterrichtssprache finden. Allerdings knüpft Thomasius mit der Bemerkung, er wolle „die Maximen des Göttlichen Rechts [...] wiederum“ zu erklären anfangen, ausdrücklich an die im Jahr zuvor gehaltene, sicher lateinische Naturrechtsvorlesung an (s. VE 3). Zudem empfiehlt Thomasius 1691 den Studenten, diverse lateinische Kompendien für die Vorlesung anzuschaffen, und kündigt an, die kurzgefassten „Theses“ der IJD zu erklären sowie die darin nicht allegierten „Autores“ mitzuteilen. Das Allegieren war die traditionelle Technik gelehrten Zitierens oder Belegens v. a. juristischer Autoritäten. Angesichts der massiven intertextuellen Bezüge zu lateinischen Textwelten außerhalb des eigenen Kollegs (mit umfangreichen lateinischen Textwiedergaben) wird die IJD-Vorlesung wohl eher auf Lateinisch als auf Deutsch gehalten worden sein.

\section{VE 6: Von denen Mängeln der Aristotelischen Ethic und von andern das Jus publicum betreffenden Sachen - Leipzig, Sommersemester 1688}

Originaltitel: „Christian Thomas Eröffnet Der Studierenden Jugend zu Leipzig/ In einem Discours Von den Mängeln der Aristotelischen Ethic, und von andern das Jus publicum betreffenden Sachen/ zwey Collegia Uber die Christliche Sitten-Lehre und über das Jus Publicum. Leipzig/ zu finden bey Moritz George

60 In der Vorlesung fand die französische wie auch die deutsche Übersetzung Verwendung, Sauters Text (s. Anm. 33) allerdings v. a. als abschreckendes Beispiel, da „des Gracians Homme de Cour so unglücklich in das Teutsche übersetzet war/ daß ich nur in der ersten Centurie meinen damaligen Auditoribus über 200. fauten zeigete/ die größten Theils die Meinung des Gracians gantz verkehrten“ (KTS 54).

61 Keine Beweiskraft kommt jedoch Thomasius’ Bemerkung zu, er habe damals ,gelehrte Dinge in der Mutter-Sprache vortragen“ wollen (KTS 53), da damit prinzipiell auch das Programma gemeint sein konnte (vgl. ${ }^{1}$ DWB s. v. vortragen, Abschn. 8).

62 Vgl. dazu KTS (393-412 u. 459) und den Codex Lectionum fol. 14 u. 43. 
Weidemannen. Halle/ Gedruckt bey Christoph Salfelden/ Chur-Fürstl. Brandenb. Hoff-BuchDr. im Hertzogth. Magdeb. 1688.“

Ausgaben: <a.> VD 17 1:050386N (48 S., 8): $\mathrm{a}_{1}=$ Landschaftsbibl. Aurich, O 2961 (3); $a_{2}=$ SB Berlin, Preuß. Kulturbesitz, Az 2784; $a_{3}=$ SLUB Dresden, Eph.lit.649-1689,2,misc.2; $\mathrm{a}_{4}=$ UB Greifswald, 523/Hf 188 adn5; $\mathrm{a}_{5}=$ ULB Halle, AB 67 13/c, 15 (7); $a_{6}$ = WLB Stuttgart, Theol.oct.15164; <b.> KTS (1701, ${ }^{21707,}$ 31721: Nr. II); <c.> KTS (1994: Nr. 2).

Literatur: Cat. II/6; Becker (1931: Nr. A/12); Lieberwirth (1955: 32) - KTS (1994: VII); Steinberg (2005: 12, 50, 62 f., 202 Nr. 12f.).

Sprache: dt.

Thomasius gibt seine Absicht bekannt, „nach Pfingsten dieses ietzigen Jahrs Vormittags üm eilff Uhr ein Collegium über die Christliche Sitten-Lehre [...] anzufangen und binnen dato und der Michaelis-Messe mit GOttes Hülfe zu absolviren“ (93f.). Für denselben Zeitraum kündigt er zudem eine Staatsrechtsvorlesung zur doctrina Juris publici an, über die er bislang nur in Privatissima gesprochen habe (111f. u. $105 \mathrm{f}$.). Die Angabe „nach Pfingsten“ bedeutet, dass Thomasius aus ungenannten Gründen, vermutlich wegen einer Reise nach Celle, von der er Samuel Pufendorf am 8. Juni berichtet (vgl. Döring 1996: Brief Nr. 163), die ersten beiden Semesterwochen übersprang und seine Kollegs erst etwas später anfangen ließ. Leider ist die Angabe nicht exakt aufzulösen. Vermutlich ist gemeint: ,nach der Pfingstvakanz‘, da die Woche zwischen dem Pfingstsonntag und Trinitatis gemäß dem kurfürstlichen Visitationsdekret von 1616 eigtl. unterrichtsfrei war (Cod. Aug. I: Sp. 917). Allerdings hat Thomasius auch im Sommersemester 1686 während der Pfingstvakanz zu lesen begonnen (s. VE 3). Die Vorlesungen dauerten also vom 4. oder 11. Juni bis Ende September 1688. Das Nachwort der KTS von 1701 ergänzt: „Dieses Programma hatte ich Anno 88. gegen Ostern publiciret“ (113), also etwa Mitte April. An anderer Stelle erwähnt Thomasius, dass sein Widersacher August Pfeiffer ,in denen Pfings-Feyertagen mein damahls ohnlängst herausgegebenes Programma“ von der Kanzel öffentlich geschmäht habe (EJH III: 129 f.). Die Vorlesungseinladung erfolgte also wohl etwa fünf Wochen vor Beginn des akademischen Jahres bzw. 7-8 Wochen vor Veranstaltungsbeginn. Zur Unterrichtssprache macht Thomasius keine Angaben, zumindest in der Ethik-Vorlesung wurde aber auf lateinaffine Unterrichtssequenzen bewusst verzichtet: Er werde ,weder einen gewissen Autor expliciren/ noch etwas in die Feder dictiren“, sondern seine Position in einem „continuirlichen discours“ darlegen (KTS 94). Zudem erklärt er im Herbst 1688 seine Absicht, für das Folgejahr nach Möglichkeit erneut eine Vorlesung zur Sittenlehre anzubieten, und zwar „mit einer deutlichen/ leichten und anmuthigen Lehrart in teutscher Sprache“ (KTS 212). Da auch die Einleitung der Sitten-Lehre, für die das Kolleg im Sommersemester 1688 „der erste 
Entwurf“ war (KTS 113), auf Deutsch verfasst ist, erscheint es nicht unplausibel, dass die Vorlesung den Übergang vom (vermutlich lateinisch unterrichteten) IJD-Kolleg des WS 1687/1688 zu einer Moralphilosophie auf Deutsch markiert.

\section{VE 7: Von den Mängeln der heutigen Academien absonderlich aber der Jurisprudenz - Leipzig, Wintersemester 1688/1689}

Originaltitel: „Christian Thomas Eröffnet Der Studierenden Jugend zu Leipzig In einem Discours Von denen Mängeln derer heutigen Academien, absonderlich aber der Jurisprudenz zwey Collegia Ein Disputatorium über seine Prudentiam ratiocinandi und ein Lectorium nach einer sonderbaren methode über die Institutiones Justinianeas. Halle/ Gedruckt bey Christoph Salfelden/ ChurFürstl. Brandenb. Hof-BuchDr. im Hertzogthume Magdeburg.“

Ausgaben: <a.> VD 17 1:050389L (47 S., 8): $\mathrm{a}_{1}=$ SB Berlin, Preuß. Kulturbesitz, Az 2784; $\mathrm{a}_{2}=$ SLUB Dresden, Eph.lit.649-1689,2,misc.3; $\mathrm{a}_{3}=$ SLUB Dresden, Jus.feud.395,misc.3; $a_{4}=$ ULB Halle, AB 67 13/c, 15 (9); $a_{5}$ = Staatl. Bibl. Neuburg a.d.D., S54/8 Enc. 106-1689; $\mathrm{a}_{6}=$ WLB Stuttgart, Theol.oct.15164; <b.> KTS (1701, ${ }^{2} 1707,{ }^{3} 1721:$ Nr. III); <c.> KTS (1994: Nr. 3).

Literatur: Cat. II/7; Becker (1931: Nr. A/13); Lieberwirth (1955: 33) - KTS (1994: VII); Steinberg (2005: 12, 48, 56, 202 Nr. 14-16).

Sprache: dt.

Thomasius kündigt zwei Veranstaltungen an, zunächst ein Logik-Disputatorium von neun Disputationes „zwischen hier und Weihnachten“, das seine eigene „Prudentiam ratiocinandi“ ([2]13) evaluieren solle, also die kurz zuvor erschienene Introductio ad Philosophiam aulicam. Die erste Disputation fand dabei am 20. Oktober bei Thomasius zu Hause statt (215). Ein vergleichbares Disputatorium gedenke er im kommenden Jahr ${ }^{63}$ abzuhalten, und zwar zum

63 Die Behauptung Steinbergs (2005: 45 u. 202 Nr. 15), diese Veranstaltung habe als Fortsetzung des ersten Disputatoriums in „der zweiten Hälfte des WS 88, also im Frühjahr 1689“ (Weihnachten bis Ostern) stattgefunden, lässt sich streng genommen nicht beweisen. Thomasius stellt ein solches Disputatorium lediglich vage für „künfftiges Jahr“ in Aussicht, macht jedoch keine näheren Terminangaben, was bei einer Veranstaltung, die noch mitten im aktuellen WS begonnen hätte, verwunderlich erscheint. Sicher ist lediglich, dass er am 9. Juni 1689 eine Vorlesung zu den IJD ankündigte (s. VE 8), deren praelectiones bereits am Folgetag begannen, die jedoch am 26. Juni wieder abgesagt werden musste (vgl. EJH III: 101-112). Im Rahmen der detaillierten Schilderung dieser Vorgänge wird kein unlängst beendetes Disputatorium zu den IJD erwähnt. Für ein solches spricht immerhin, dass Thomasius am 22. 12. 1688 Pufendorf von einem bevorstehenden Kolleg berichtete, worauf dieser für das „gemeldete Collegium“ gutes Gelingen wünscht (BW Nr. 97). Das Thema ist im Antwortbrief nicht erwähnt; relevant 
Naturrecht (217). Weiterhin lädt Thomasius zu einem privatrechtlichen Lectorum über die Institutiones Iustiniani ein, das ,zwischen dato und der Oster Messe künfftiges Jahrs absolviret werden“ könne (230 u. 232). Interessenten werden gebeten, sich „,auff den Montag nach der Zahlwoche“ bei ihm einzufinden. Das Lectorum war somit für die Dauer vom 15. Oktober 1688 bis zum Semesterende geplant. Angekündigt wurde es „,in der Michaelis Woche. 1988“ (= 30. 9-7. 10.), ${ }^{64}$ d. h. ein bis zwei Wochen vor Veranstaltungsbeginn.

\section{Corrigendum: Invitationes ad auscultandas Disputationes super Huberi Prælectionibus - Leipzig, Sommersemester 1689}

Ein solches Programm zu den Prælectiones des Juristen Ulrik Huber wird von allen Werkverzeichnissen angeführt. ${ }^{65}$ Steinberg (2005: 57 u. 203 Nr. 18) setzt unter Berufung darauf für das Sommersemester 1689 eine „Disputierübung zum Privatrecht der Institutionen und Pandekten nach Huber, Praelectionum tomi tres“ an. Diese sei im Voraus durch die Invitationes angekündigt worden. ${ }^{66}$ Ein solches Werk ist indes nicht nachweisbar und auch gar nicht wahrscheinlich. Ausgangspunkt für den populären Irrtum ist ein entsprechender Eintrag im Catalogus scriptorum (Ausgabe 1711). Dort findet sich unter den „Programmata aliaque scripta“ zwischen Einträgen zu 1689 und 1690 folgende Mitteilung: „Invitationes ad auscultandas Disputationes super Huberi Prælectionibus. Adjunctæ jam sunt editioni Lipsiensi Positionum Huberi“. Im Gegensatz zu den Programmata, die im Catalogus originaliter zitiert werden (mit Erscheinungsjahr und z. T. Buchformat), wird bezüglich dieser Invitationes also lediglich auf eine Leipziger Ausgabe der „Positiones“ verwiesen (in anderen Auflagen des Catalogus: der „Prælectiones“). Damit kann nicht die Ausgabe von Hubers Praelectiones iuvris civilis gemeint sein, die Thomasius 1707 in Leipzig verantwortet hatte. ${ }^{67}$ Der fragliche Eintrag findet sich nämlich bereits in der

wäre für Pufendorf aber wohl nur eine Mitteilung über eine naturrechtliche Veranstaltung gewesen.

64 So auch in EJH III (30): ,als ich 7) an vergangener Michaelis-Messe meine Introduction ad Philosophiam Aulicam heraus gegeben, und ein Collegium Disputatorium darüber angeschlagen“.

65 Vgl. Zedler (Bd. 43: Sp. 1594, d), Becker (1931: 520 Nr. 16), Lieberwirth (1955: Nr. 40) und Steinberg (2005: 12).

66 Das Literaturverzeichnis bei Steinberg (2005: 223) weist die angeblichen Invitationes sogar mit der Angabe „Halle 1689“ aus (bei Becker und Lieberwirth dagegen s. l. und „Ohne Jahr“). 67 Darin kommt er kurz auf Leipziger Disputationen zu sprechen, allerdings solche des Jahres 1684: „Postea cum Anno 1684. præfatas Positiones Huberianas in Academia Lipsiensi publicis Disputationibus iterum ventilare instituissem [...]“ (Thomasius 1707: I: Praefatio). 
Erstauflage des Catalogus von 1693. Er ist dort zwischen zwei Programmschriften von 1687 (= VE 4 u. 5) platziert und noch mit „3. \& 4.“ eingeleitet - es geht folglich um zwei separate Invitationes. In Wahrheit liegt eine Verwechslung mit den beiden Programmata von 1683 und 1684 vor, die 1684/1685 tatsächlich Thomasius' älterer Huber-Ausgabe beigegeben worden waren (s. VE 1 u. 2). ${ }^{68}$ Diese beiden Programmschriften sind die fraglichen Invitationes ad auscultandas Disputationes super Huberi Prælectionibus. Die Verwendung des Temporaladverbs („Adjunctæ jam sunt“) erklärt sich aus dem Umstand, dass VE 1/2 im Catalogus erst nach den Programmschriften von 1686 und 1687 eingeordnet wurden, obwohl sie eigentlich älter sind. Folglich hat es eine entsprechende Disputierübung im Sommer 1689 nicht gegeben.

\section{VE 8: Lectiones gratuitæ de differentiis justi \& decori (bzw. IJD) - Leipzig, Sommersemester 1689}

Originaltitel: unbekannt

Ausgaben: <a.> -; <b.> EJH III $\left({ }^{1} 1721,{ }^{2} 1724: 101\right)$.

Literatur: -

Sprache: lat.

Eine in den Werkverzeichnissen übersehene Vorlesungseinladung zum Sommersemester 1689 findet sich in EJH III (101). ${ }^{69}$ Am So., dem 9. Juni, fünf Wochen nachdem der Leipziger Theologe August Pfeiffer seine gegen Thomasius gerichtete Atheismus-Vorlesung begonnen hatte (s. Anm. 37), veröffentlicht Thomasius ein lateinisches „Programma“, in dem er verkündet, gleich ab dem nächsten Tag ein Semester lang über die IJD zu lesen, und zwar zwischen 16 und 17 Uhr, d.h. exakt zur selben Zeit wie Pfeiffer, was einer Provokation gleichkam (vgl. allg. Horn 1897: 21 f.). Die Verteidigung seines guten Rufs verlange es, dass er zunächst unentgeltlich einige einleitende Lektionen vorausschicke (EJH III: 101). Darin werde er die Grundlagen „de juste \& Decoro“ erklären, aber auch „de Natura Atheismi“ sprechen und die eigenen Positionen gegen die ruchlosen Vorwürfe seines Widersachers (,immodestas \& impias malevoli cujusdam calumnias“) verteidigen. Obwohl die Gegenseite dieses Programma unverzüglich vom Schwarzen Brett abreißen lässt und gleich am folgenden Vormittag ein (juristisch nicht bindendes) Mandat des Rektors gegen

68 Die Ausgabe erschien in Leipzig und Frankfurt bei Weidmann; der Catalogus selbst verkürzt den Eintrag zu: „Lipsiæ apud Mauritium Georgium Weidemannum“ (Nr. III/2).

69 Zum Inhalt der Vorlesung vgl. EJH III (104-108), zum Stoff der tatsächlich absolvierten Sitzungen EJH III (135f.). 
die angekündigte Vorlesung erwirkt, beginnt Thomasius vor einer offenbar beachtlichen Zuhörerschaft wie geplant zu lesen (102-104). Bereits im Verlauf der ersten beiden Vorlesungswochen wird ihm jedoch mit Befehl des Oberkonsistoriums in Dresden die Abhaltung der Veranstaltung untersagt (108f.). Da sich die Anordnung allerdings nicht ausdrücklich auch auf die „lectiones praeliminares“ bezieht, führt Thomasius diese fort, bis ihm auch dies am 13. Juli explizit untersagt wird (134-136). Als es seinen Gegnern nach fünf Wochen schließlich gelingt, die ohnehin nur auf einige Wochen konzipierte Vorlesung zu unterbinden, wandelt Thomasius sein Kolleg am 15. Juli in Lectiones gratuitas de praejudiciis um, die Mo./Do. zu späterer Stunde (17-18 Uhr) auf Deutsch gehalten wurden. ${ }^{70}$ Diese Vorlesung findet im Wintersemester eine Fortsetzung (vgl. EVL 24) und endet schließlich vorzeitig mit dem Unterrichtsverbot im März 1690.

Die äußerst kurzfristige Ankündigung der Vorlesung durch VE 8 ist zweifellos dem eskalierenden Konflikt mit der Theologischen Fakultät geschuldet. Der späte Beginn verdankt sich allerdings einer für Ende April geplanten ${ }^{71}$ Reise nach Berlin und Hamburg, bei der Thomasius u. a. Samuel Pufendorf treffen wollte und von der er wohl erst Ende Mai zurückkehrte (EJH III: 99f.). Da das akademische Jahr bereits am 6. Mai begonnen hatte, war ein späterer Unterrichtsbeginn im Sommersemester 1688 also eingeplant.

Nicht sicher zu klären ist, ob es sich bei VE 8 um einen chirographischen oder typographischen Text handelte: Angesichts der sich überstürzenden Ereignisse und des geringen Textumfangs wird man zunächst an einen handschriftlichen Zettel denken. Thomasius selbst spricht mehrfach von einem Programma und einmal von einem Zettul, Pfeiffer von einer schedula (102-104). Da auch der Dekan der Philosophischen Fakultät VE 8 als programma (und ausdrücklich nicht als scheda) bezeichnet, ist eine gedruckte Vorlesungseinladung jedoch nicht auszuschließen (vgl. Prinz 2019). In welcher Sprache die lectiones praeliminares gehalten wurden, bleibt ebenfalls unerwähnt - die Inhaltszusammenfassung in EJH III (104-108) gibt Thomasius jedenfalls auf Deutsch. Dass er im Hinblick auf die Ersatzvorlesung De praejudiciis dann allerdings erklärt, diese werde „aus gewissen Ursachen in Teutscher Sprache“ gehalten (VGH III: 628, vgl. auch 761), spricht eher für einen lateinischen Vortrag der Präliminarvorlesung.

70 In VGH III (Nr. VII: § II ff.) liefert Thomasius „die summam und Haupt-Puncte desselbigen“, wie er sie „vorher pro memoria aufs Papier entworffen“ hatte. Auf diesem Weg fand die Vorlesung schließlich Eingang in die Werkverzeichnisse (Becker 1931: A/20; Lieberwirth 1955: 43). 71 Pufendorf nimmt bereits am 10. April in einem Brief an Thomasius auf ein allerdings noch nicht genau geplantes Treffen in Berlin Bezug (vgl. Döring 1996: Nr. 163). 


\section{VE 9: Vorschlag wie ein junger Mensch zu einem honnéten und galanten Leben zu informiren sey - Leipzig, Wintersemester 1689/1690}

Originaltitel: „Christian Thomas Eröffnet Der Studierenden Jugend Einen Vorschlag/ Wie er einen Jungen Menschen/ der sich ernstlich fürgesetzt/ GOtt und der Welt dermahleins in vita civili rechtschaffen zu dienen/ und als ein honnet und galant homme zu leben/ binnen dreyen Jahre Frist in der Philosophie und singulis Jurisprudentiæ partibus zu informiren gesonnen sey. Halle/ Gedruckt und verlegt von Christoph Salfelden/ Chur-Fürstl. Brandenb. Hoff- und RegierungsBuchdrucker. Anno 1689.“

Ausgaben: <a.> VD 17 14:008886H (47 S., 8): a $_{1}=$ UB Augsburg, 02/VI.2.8.111; $\mathrm{a}_{2}=$ Landschaftsbibl. Aurich, O 2961 (4); $\mathrm{a}_{3}=$ SB Berlin, Preuß. Kulturbesitz, Az 2784; $a_{4}=$ SB Berlin, Preuß. Kulturbesitz, Az 2788; $a_{5}=$ SLUB Dresden, 3.A.10027,angeb.2; $a_{6}=$ ULB Halle, AB 67 13/c, 15 (8); $a_{7}=$ Staatl. Bibl. Neuburg a.d.D., S54/8 Enc. 106-1689; $a_{8}=$ LB Oldenburg, LIT II 2 373,3; $a_{9}=$ WLB Stuttgart, Theol.oct.15164; <b.> KTS (1701, ${ }^{21707,}{ }^{3} 1721$ : Nr. IV); <c.> KTS (1994: Nr. 4). Literatur: Cat. II/8; Becker (1931: Nr. A/17); Lieberwirth (1955: 36) - KTS (1994: VII f.); Steinberg (2005: 12, 28-31 [dort irrtümlich als „Ankündigung für das SS 89“], 48 f., 203 Nr. 19).

Sprache: dt.

Am 10. Juni, nur einen Tag nachdem Thomasius als Reaktion auf die Pfeiffersche Atheismus-Vorlesung ein lateinisches Programm für das bereits laufende Sommersemester (s. VE 8) publiziert hat, kündigt er „mitten in diesen troublen" (EJH III: 162) für das Wintersemester ein eigenes umfassendes Studienkonzept an, welches in einem dreijährigen Zyklus weite Teile des philosophischen und juristischen Curriculums abdecken solle, und zwar in folgender thematischer Progression: Logik, Geschichte, praktische Philosophie (Ethik inkl. Naturrecht, Politik, Ökonomie, Decorum/Galanterie), Oratorie, Rechtsauslegung, Privatrecht, Lehnrecht, öffentliches Recht und Kirchenrecht. Er werde täglich zwei Stunden unterrichten und den Anfang nach der Michaelismesse machen (KTS 267), also am 14. Oktober 1689, 18 Wochen nach der Ankündigung durch VE 9. Das geplante Studienkonzept war modular aufgebaut. Den Teilnehmern war es freigestellt, am Ende eines Semesters „nach Gefallen ab und zuzutreten“, die zweite Stunde konnte überdies fakultativ belegt werden. ${ }^{72}$

72 Vgl. EVL (3f., 20-22). Dass es sich dabei um die Leipziger Vorlesungsreihe handelte, geht auch aus dem Nachwort hervor („Erinnerung des Autoris an den Leser“): Thomasius habe die EVL im Herbst 1689 „zu verfertigen und darüber zu lesen angefangen“. Im Februar 1690 sei der Text bis auf das letzte Kapitel bereits gedruckt gewesen. Aufgrund der Flucht aus Leipzig 
Tatsächlich konnte dieses ambitionierte Vorhaben zunächst plangemäß begonnen werden; es fand durch das Lehrverbot im März 1690 jedoch ein vorzeitiges Ende (vgl. KTS 270).

\section{VE 10: Primum Programma Halense de instituendis lectionibus publicis \& privatis, Philosophicis \& Juridicis. 1690 - Halle, Sommersemester 1690}

Originaltitel: „Auspice Divina Gratia Et Jussu Serenissimi \& Potentissimi Electoris Brandenburgici \&c. \&c. Christianus Thomasius, JCtus, \& Ipsius Serenitatis Electoralis Consiliarius, Scholas Philosophicas \& Juridicas, publicas \& privatas, Halæ Saxonum Post festum Pentecostes currentis anni 1690. aperiendas publicè indicit.“

Ausgaben: <a.> VD 17 12:185585Z ([6] Bl., 2º): $\mathrm{a}_{1}=$ SB Berlin, Preuß. Kulturbesitz, 4“ Np 18020; $\mathrm{a}_{2}=$ Forschungsbibl. Gotha, Phil $2^{\circ}$ 00095/01 (05); ${ }^{\star} \mathrm{a}_{3}=\mathrm{ULB}$ Halle, Pon Yb 3835, FK (Verlust 1947); $\mathrm{a}_{4}=$ KB Kopenhagen, 9, 84p 00071; $\mathrm{a}_{5}=$ BSB München, 2 Diss. 13 (Beibd. 37); <b.> PT (1724: Nr. VI, S. 101-117); <c.> PT (2010: Nr. VI).

Literatur: Cat. II/9; Becker (1931: Nr. A/21); Lieberwirth (1955: 45) - Steinberg (2005: 12, 69 f., 76, 203 Nr. 21); PT (2010: xiii f.).

Sprache: lat.

Am Sonntag Quasimodogeniti (27. April 1690), kurz nach der Übersiedlung nach Halle und Bestallung als Kurfürstlicher Rat und Professor, publiziert Thomasius sein Primum Programma Halense. Mit diesem knüpft er an das Studienkonzept vom Vorjahr (VE 9) an, welches noch einmal referiert wird, allerdings auf Lateinisch. Gründe für die Sprachwahl werden nicht genannt. Vermutlich war gerade an einer noch nicht als Universität etablierten Hohen Schule wie Halle die Kontextualiserung der Einladung in einem gelehrten lateinischen Rahmen ein Signal akademischer Ambition. Thomasius kündigt zudem an, den in Leipzig begonnenen Vorlesungszyklus abzubrechen und in Halle, wo er nun auch Lectiones publicae anbieten konnte, erneut ,ab ovo“ $\mathrm{zu}$ beginnen (PT $110 \mathrm{f}$.). Die Unterrichtsvorbereitung erfolgte angesichts der Umstände unter großem Zeitdruck, zumal die Vorlesungstätigkeit bereits am 16. Juni, dem Mo. nach Trinitatis, beginnen sollte (VGH II: 73, 118 u. 283). Der Lehrzyklus in Halle erwies sich als großer Erfolg. Obwohl Thomasius nach eigenem Bekunden nicht versuchte, Leipziger Studenten abzuwerben - er „notificirte nur seine Ankunfft erst privatim seinen Auditoribus privatissimis [...] hernach publicè durch sein

habe die für die Ostermesse geplante Veröffentlichung dann auf die Neujahrsmesse 1691 verschoben werden müssen. 
Programma [...] jederman“ -, seien über 50 Zuhörer zur ersten Sitzung erschienen (Summ. Anzeige $257 \mathrm{f}$.). Möglich war dies wohl nur wegen der relativ frühzeitigen Vorlesungsankündigung, sieben Wochen vor Veranstaltungsbeginn. Die eigentliche Einladung zu den öffentlichen Lektionen (mit Ort und Zeit) erfolgte dann 15 Tage vor der ersten Sitzung (s. VE 11). Eine Bestätigung und Ergänzung erfährt diese Darstellung durch einen kurzen Text, der von der Forschung bislang völlig übersehen wurde: Datiert auf den 20.11.1690 und mit der Ortsangabe Halle erschien ohne Hinweis auf Drucker oder Verleger eine vierseitige Schrift mit dem Titel:

Copey eines Schreibens/ Welches ein in Halle sich befindender Studiosus an einen guten Freund in Breßlau von dem Zustand der in Halle auffzurichtenden Neuen Academie abgehen lassen. ${ }^{73}$

Der Autor, angeblich ein „F.J.R.“, beschreibt darin anschaulich die Studienbedingungen im Jahr 1690. Für die geplante Universitätsgründung in Halle habe man an Ostern Thomasius berufen, damit dieser „mit Lectionibus Philosophicis und Juridicis den Anfang machen solle“. Thomasius habe ein öffentliches Programma (= VE 10) vorgelegt und bald eine „nicht zu verachtende Zahl der studierenden Jugend“ angezogen, die bei ihm seit Trinitatis studiert hätte und für die er auch die Jurisdiktion zugesprochen bekommen habe. Neben den öffentlichen Vorlesungen erwähnt die Copey ein bislang unbekanntes Privatissimum, in dem Thomasius mit einigen auf Empfehlung hin ausgewählten Studenten „das/ was sie publicè gehöret/ continuô examine repetiret“ habe. Dieses Kolleg sei im Wintersemester fortgesetzt worden. Der Autor der Copey bemüht sich merklich, sowohl Lehrende als auch Studenten für Halle anzuwerben: Interessierte Doctores und Magistri dürften hier, sofern sie „die Confidenz zu ihren studiis tragen/ daß sie Collegia halten und dozieren können“, dies „frey und unverboten“ tun, Nicht-Graduierte müssten zuerst in Magdeburg eine Genehmigung einholen. Den Studenten wird das verfügbare Angebot an Exercitia ${ }^{74}$ und das frankophone Element ${ }^{75}$ in der Stadt angepriesen und die

73 VD 17 23:715652C; erhaltene Exemplare in: KB Kopenhagen, 9, 84p 00071 und HAB Wolfenbüttel, Pd $4^{\circ} 45$ (2).

74 So besitze der Stallmeister v. Berghorn eine große Zahl gut abgerichteter Pferde und die Sprach- und Exerzitienschule von M. la Fleur biete die Möglichkeit Fechten, Tanzen, Französisch und Italienisch zu lernen (zu diesen vgl. Schrader 1894, II: 354-356 und Schröder 2017: 40).

75 Aufgrund der vielen hugenottischen Flüchtlinge in der Stadt werde „sowohl in Frantzösischer als teutscher Sprache geprediget“. Man könne sich leicht im Französischen perfektionieren und habe „eine freye und wohlanständige conduite in Gesellschafft bey ihnen/ ohne kostbahres reisen“. 
zu erwartenden Lebenshaltungskosten aufgeschlüsselt. Der Ort sei „sehr lustig an der Saale gelegen/ und mit vielen artigen Gärten/ Wiesen und Dörffern umbgeben.“ Der deutliche Werbecharakter der Copey in Verbindung mit ihrem sprachlichen Duktus (,gute und honnête Conversation“, „wohlanständige conduite“) und der reichlich unplausiblen Entstehungsbehauptung legt die Vermutung nahe, dass die Schrift von Thomasius selbst verfasst wurde. ${ }^{76}$

\section{VE 11: Programma lectiones illas determinans - Halle, Sommersemester 1690}

Originaltitel: „Q.D. B. V. Christianus Thomasius, JCtus \& Sereniss. ac Potentiss. Elect. Brandenburgici Consiliarius, Philosophiæ \& Jurisprudentiæ Cultoribus Lectionum publicarum non ita pridem publicè promissarum auspicium determinat.“

Ausgaben: <a.> VD 17 12:185593R ([2] Bl., 2º): $\mathrm{a}_{1}=$ Bibl. der Franckeschen Stiftungen Halle, 179 C 9 [9]; $\mathrm{a}_{2}$ = BSB München, 2 Diss. 13 (Beibd. 46); <b.> PT (1724: Nr. VII, S. 118-124); <c.> PT (2010: Nr. VII).

Literatur: Cat. II/10; Becker (1931: Nr. A/22); Lieberwirth (1955: 46) - Steinberg (2005: 13, 69, 76, 203 Nr. 21); PT (2010: xiv).

Sprache: lat.

Am 1. Juni, exakt fünf Wochen nach VE 10, bekräftigt Thomasius seine Absicht, in Halle Vorlesungen zu halten. Die organisatorischen Belange sind nun so weit geklärt, dass er mittlerweile Ort und Zeit für die Lectiones publicas benennen kann. Diese würden am 16. Juni, also nach der Pfingstvakanz, um 11 Uhr beginnen (,die Lunæ post instans Festum Trinitatis horâ antemeridianâ undecimâ“), und zwar „in ædibus Weichartianis“, dem sog. Weichartschen Haus gegenüber der Ulrichskirche (zu diesem: Neuß 1931: 463).

\section{VE 12: Programma de Collegio privato in Institutiones Justinianeas - Halle, Wintersemester 1690/1691}

Originaltitel: Q.F.F. Q. S. Christianus Thomasius, JCtus \& Consiliarius Electoralis Brandenburgicus. Cupidæ Legum Juventuti Collegium privatum super Institutionibus Justinianeis, Novâ Methodo Halæ Saxonum, d. XX. Octobris horâ 10. andemerid. currentis Anni inchoandum publicè notum facit.

76 Zudem hat sich das Kopenhagener Exemplar der Copey in einem Sammelband mit lauter Programmschriften von Thomasius aus den Jahren 1690-1693 erhalten. 
Ausgaben: <a. > KB Kopenhagen, 9, 84p 00071; <b.> PT (1724: Nr. VIII, S. 125133); <c.> PT (2010: Nr. VIII).

Literatur: Cat. II/12; Becker (1931: Nr. A/23); Lieberwirth (1955: 49) - Steinberg (2005: 13, 81 f., 203 Nr. 22, 24); PT (2010: xv).

Sprache: lat.

Am 28. Sept. 1690 (in der Literatur z. T. irrtümlich: 5. 10.), dem 15. Sonntag nach Trinitatis, kündigt Thomasius zusätzlich zu seinem öffentlichen cursus Philosophiæ \& Juris (VE 10 \& 11) ein Privatkolleg über Justinians Institutiones an. Dieses werde am 20. Oktober, also drei Wochen später, um 10 Uhr vormittags beginnen und voraussichtlich bis Ostern 1691 abgeschlossen sein. 

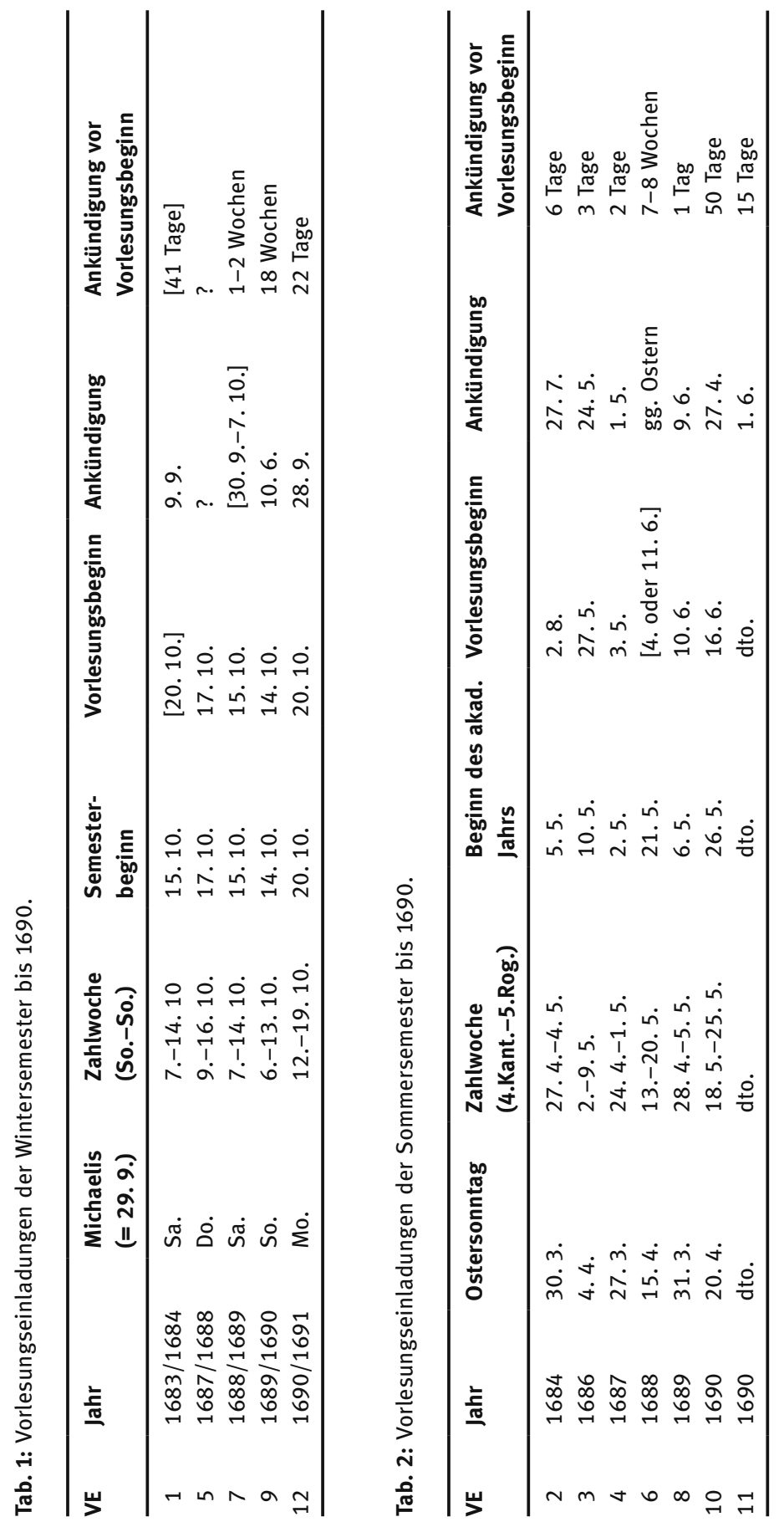


\section{Fazit}

Die Auswertung der Datierungspraxis in Christian Thomasius' Programmschriften bis 1690 lässt Folgendes erkennen (s. Tab. 1 und 2):

1. In seinen Kollegs orientierte sich Thomasius vorrangig am Beginn des Wintersemesters. Während er im Herbst stets pünktlich zum offiziellen Semesterbeginn zu lesen anfing, trifft dies für das Sommersemester lediglich einmal zu. Das ist erstaunlich, da man die wesentliche Zäsur eigentlich im Frühjahr, am Sonntag Rogate, mit dem das akademische Jahr begann, erwarten sollte. Huttner (2007a: 205) hat zu zeigen versucht, dass im 17. Jahrhundert nicht das Semester, sondern das akademische Jahr die „den äußeren Ablauf des universitären Lehrbetriebs maßgebliche strukturierenden Zeiteinheit“ gebildet habe. Offenbar hatte Thomasius jedoch seinen eigenen Zeitplan. Der relevante Fixtermin im akademischen Jahresverlauf war für ihn der Beginn des Wintersemesters, an Rogate orientierte er sich nur lose.

2. Die Vorlesungseinladung erfolgte in der Regel kurzfristig, im Normalfall etwa zwei Tage bis vier Wochen vor Veranstaltungsbeginn. Schwankungen erklären sich dabei z.T. aus externen Faktoren (Reisen, Konflikten etc.). Einen Sonderfall, der sich jedoch nicht auf das Gracián-Kolleg übertragen lässt, stellt das ambitionierte Studienkonzept von 1689 dar, dessen Planung offenbar einen längeren Vorlauf erforderte und das bereits während des laufenden Sommersemesters für den Winter angekündigt wurde. Thomasius merkt an, dass er „einen gewissen numerum von Auditoribus“ nicht zu überschreiten gedenke und Interessenten „dieserwegen beyzeiten bey mir sich anzugeben belieben wollen“ (KTS 267). Die frühe Einladung barg jedoch auch Risiken, indem sie in der sich zuspitzenden Leipziger Konfliktgeschichte den anderen Parteien ausreichend Zeit zur Einleitung von Gegenmaßnahmen verschaffte. So konnte etwa August Pfeiffer zwischenzeitlich sein Wohlmeinendes Gutachten veröffentlichen, mit dem er, wenngleich erfolglos, versuchte, Thomasius „die Auditores abspänstig zu machen“ (KTS 270).

3. Thomasius hat im Untersuchungszeitraum nie ein ganzes Semester im Voraus, also etwa vor Beginn des akademischen Jahrs für das Wintersemester, zu einem Kolleg eingeladen. Mitunter wird eine längerfristige Lehrplanung beiläufig angedeutet; ${ }^{77}$ es handelt sich dabei jedoch um unverbindliche Absichtserklärungen, nicht um durch Angaben zu Zeit und Ort substanziierte konkrete Vorlesungseinladungen.

77 So erwähnt etwa das Herbst-Programma 1688 eine mögliche Vorlesung zur Sittenlehre für das Folgejahr (VE 7; KTS 212). 
Hinzu kommt, dass Rezeptionszeugnisse für VE 5 nicht vor dem Herbst 1687 nachzuweisen sind. Das älteste stammt von einem namentlich nicht genannten ,auswärtigen“ Freund, der das von Thomasius übersandte GraciánProgramma in seinem Umfeld bekannt gemacht hatte und die Reaktionen darauf - von anonymen „gelehrten Freunden“ - wiederum Thomasius übermittelte (KTS 56-70). Dieser beantwortete die Briefe bereitwillig - die lateinischen auf Latein, die französischen jedoch auf Deutsch. Diese Schreiben bieten wenig Anhaltspunkte für eine nähere Bestimmung. Die Bezeichnung des Deutschen als „notre langue“ (KTS 59) verweist jedenfalls auf eine Region des deutschen Sprachgebiets, die von Thomasius in seiner Antwort mit der erweiterten Präpositionalphrase „bey ihnen [= Adressat] drunten“ (KTS 65) deiktisch perspektiviert wird. Unter diesen Schreiben findet sich auch ein datierter lateinischer Briefauszug („Extr. lit. Fratris mei Abbatis“), in dem das „programma \& germanicum discursum“ kommentiert ist. Bei der Angabe „23. Octobr. 1687“ dürfte es sich jedoch angesichts der Nennung des „Fratris mei Abbatis“, den Thomasius in seiner Antwort stets als Reverendus Abbas tituliert, um eine Datierung aus dem Geltungsbereich des neuen Kalenders gehandelt haben. Der Brief wurde also vermutlich am 13. Oktober (alten Stils) aufgegeben, dem Donnerstag vor Vorlesungsbeginn.

Zudem hat sich in der Uffenbach-Wolfschen Briefsammlung in Hamburg eine Rechnung des Leipziger Buchhändlers Moritz Georg Weidmann erhalten, ${ }^{78}$ die belegt, dass Thomasius am 19. Juli 1687 zwei unterschiedliche Ausgaben (frz. und dt.) des Handorakels bestellte. Anders als Weißenborn (1931: 434) annimmt, wird diese Bestellung - ein Vierteljahr vor Vorlesungsbeginn - kaum für die Studenten gedacht gewesen sein. Vermutlich fing Thomasius Ende Juli mit der Planung oder Vorbereitung der Vorlesung für das Wintersemester an.

Damit bleibt festzuhalten: Thomasius hat seine berühmte akademische Programmschrift im Herbst 1687 vorgelegt, vermutlich irgendwann im September. Die erste Sitzung des Gracián-Kollegs fand dann am Montag, dem 17. Oktober (alten Stils), um 9 Uhr morgens statt. Keine dieser Begebenheiten ereignete sich also am Reformationstag. Die 130 Jahre alte Heldenerzählung vom akademischen language shift als einer antilateinischen Reformation der Wissenschaftsdomäne muss damit auf ihr zentrales Motiv verzichten. Einmal mehr zeigt sich: „Wie auf vielen großen Erzählungen der Geschichtswissenschaft so lastet gerade auch auf jenen der Universitätsgeschichtsschreibung vielfach immer noch das Erbe des 19. Jahrhunderts“ (Füssel 2015: 59).

78 Hamburg, Staats- und Universitätsbibliothek, Sup. ep. (4º) 33, Bl. 14. Erwähnt bzw. gedruckt bei Weißenborn (1931: 433-437), Lehmstedt (1992: 339-350) und BW (Nr. 198). 


\section{Quellen und Literatur}

\section{Handschriften/Archivalien}

Codex Lectionum $=$ Halle, Universitäts- und Landesbibliothek, Yb 3885c, $2^{\circ}$

Dresden, Sächsisches Hauptstaatsarchiv, 10026 Geh. Kabinett Loc. 00554/021 („Des

Dr. Thomasii zu Halle Vocation betr. ao. 1705. 1709.“)

Hamburg, Staats- und Universitätsbibliothek, Sup. ep. (4) 33

Hamburg, Staats- und Universitätsbibliothek, Sup. ep. 51

Leipzig, Universitätsarchiv, Rektor, Rep. 01/05/011

\section{Werke von Christian Thomasius}

BW = Grunert, Frank, Matthias Hambrock \& Martin Kühnel (Hrsg.) (2017): Christian

Thomasius. Briefwechsel. Historisch-kritische Edition. Bd. 1. Berlin, Boston: de Gruyter.

Cat. $=$ [Thomasius, Christian 1693]: Catalogus Scriptorum publicè editorum Christiani Thomasii, JCti. Halle: Salfeld.

EJH III = Thomasius, Christian (1724): Ernsthaffte, aber doch Muntere und Vernünfftige Thomasische Gedancken $u$. Erinnerungen über allerhand außerlesene Juristische Händel. Dritter Theil. Zweyte Auflage. Halle: Rengerische Buchhandlung.

$\mathrm{EVL}=$ Thomasius, Christian (1691): Einleitung zu der Vernunfft-Lehre/ Worinnen durch eine leichte/ und allen vernünfftigen Menschen/ waserley Standes oder Geschlechts sie seyn/ verständliche Manier der Weg gezeiget wird/ ohne die Syllogisticâ das wahre/ wahrscheinliche und falsche von einander zu entscheiden/ und neue Warheiten zu erfinden. Halle: Christoph Salfeld.

IJD = Thomasius, Christian (1688): Institutiones Jurisprudentiæ Divinæ, in Positiones succinctè contractæ, In quibus Hypotheses Illustris Pufendorffii circa doctrinam Juris Naturalis Apodicticè demonstrantur \& corroborantur [...] Frankfurt, Leipzig: M. G. Weidmann.

KTS = Thomasius, Christian (1701): Allerhand bißher publicirte Kleine Teutsche Schrifften/ Mit Fleiß colligiret und zusammen getragen; Nebst etlichen Beylagen und einer Vorrede. Halle: Christoph Salfeld.

Positiones sive Lectiones = Ulrici Huberi Positiones sive Lectiones Juris Contractæ secundum Institutiones [\& Pandectas] ad primordia disciplinæ usumq; seculi adtemperatæ \& in Academia Lipsiensi publicè iterum ventilatæ ac scholiis nonnullis auctæ à Christiano Thomasio. Bd. I (1685). Bd. II (1684). Leipzig, Frankfurt: Weidmann.

PT (1724) = Programmata Thomasiana, et alia scripta similia breviora coniunctim edita, cum notis hinc inde de noco adiectis. Halle, Leipzig: Johann Christoph Krebs.

PT (2010) s. Steinberg (2010).

RG = Thomasius, Christian (1709): Drey Bücher der Göttlichen Rechtsgelahrtheit [...] Halle: Renger. [= deutsche Übersetzung der IJD]

Summ. Anzeige = Thomasius, Christian (1696): Summarische Anzeige und kurtze Apologie, wegen der vielen Anschuldigungen und Verfolgungen/ damit Ihn etliche ChurSächsische Theologen zu Dresden/ Wittenberg und Leipzig nun etliche Jahr her beleget und diffamiret. In: Das Recht Evangelischer Fürsten In Theologischen Streitigkeiten/ gründlich ausgeführet/ und wider die Papistischen Lehrsätze eines Theologi zu Leipzig 
vertheydiget von D. Christian Thomasen/ P.P. und Lic. Enno Rudolph Brenneysen. Halle: Christoph Salfeld, [239]-288.

[Thomasius, Christian (,F.J.R.“)] (1690): Copey eines Schreibens/ Welches ein in Halle sich befindender Studiosus an einen guten Freund in Breßlau von dem Zustand der in Halle auffzurichtenden Neuen Academie abgehen lassen. [Halle].

Thomasius, Christian (1707): Ulrici Huberi Prælectionum juris civilis tomi tres, secundum Institutiones et Digesta Justiniani. accedunt huic editioni novæ additiones Christiani Thomasii [...] Leipzig: Gleditsch.

Thomasius, Christian (1717): D. Melchiors von Osse Testament gegen Hertzog Augusto, Churfürsten zu Sachsen [...] Halle: Rengerische Buchhandlung.

$\mathrm{VE}=$ Vorlesungseinladung (s. oben)

VGH II/III = Thomasius, Christian (1724/1725): Vernünfftige und Christliche aber nicht Scheinheilige Thomasische Gedancken Und Errinnerungen Uber allerhand Gemischte Philosophische und Juristische Händel. Andrer/Dritter Theil. Halle: Rengerische Buchhandlung.

\section{Literatur}

Academia Lipsiensium Academica Oder Leipziger Universitäts-Geschichte / Welche Den gegenwärtigen Zustand der Academischen Gelehrsamkeit auf der Universität Leipzig sorgfältig eröffnen. Der Fünffte Theil (1723). Leipzig: Joh. Christoph Coerner.

Achermann, Eric (2003): Substanz und Nichts. Überlegungen zu Baltasar Gracián und Christian Thomasius. In: Manfred Beetz \& Herbert Jaumann (Hrsg.), Thomasius im literarischen Feld. Neue Beiträge zur Erforschung seines Werkes im historischen Kontext (Hallesche Beiträge zur Europäischen Aufklärung 20). Tübingen: Niemeyer, 7-34.

Adamzik, Kirsten (2016): Textlinguistik. Grundlagen, Kontroversen, Perspektiven. 2., völlig neu bearb., aktualis. u. erw. Neuaufl. Berlin, Boston: de Gruyter.

Amelot de la Houssaie, Nicolas (1684): L' Homme De Cour. Traduit de l'Espagnol de Baltasar Gracian. Par le Sieur Amelot de la Houssaie. Avec des notes. Paris: Veuve Martin, \& Jean Boudot.

[Anonym] (1751): Fortgesetzte Nachrichten von dem Fortgang und Ausnehmen der Berlinischen Kunst- und Werck- oder Realschule, mit Anmerckungen. In: Leipziger Sammlungen von Wirthschafftlichen, Policey-Cammer- und Finantz-Sachen 7, 675-725.

Bach, Thomas, Jonas Maatsch \& Ulrich Rasche (Hrsg.) (2008): „Gelehrte“ Wissenschaft. Das Vorlesungsprogramm der Universität Jena um 1800 (Pallas Athene 26). Stuttgart: Franz Steiner.

Becker, Walter (1931): Thomasius-Bibliographie. In: Max Fleischmann (Hrsg.), Christian Thomasius. Leben und Lebenswerk (Beiträge zur Geschichte der Universität HalleWittenberg 2). Halle/S.: Niemeyer, 511-553.

Blanke, Horst Walter (1983): Bibliographie der in periodischer Literatur abgedruckten Vorlesungsverzeichnisse deutscher Universitäten. Teil 1. In: Berichte zur Wissenschaftsgeschichte 6, 205-227.

Blanke, Horst Walter (1987): Bibliographie der in periodischer Literatur abgedruckten Vorlesungsverzeichnisse deutscher Universitäten. Teil II. In: Berichte zur Wissenschaftsgeschichte 10, 17-43.

Blanke, Horst Walter (1988): Bibliographie der in periodischer Literatur abgedruckten Vorlesungsverzeichnisse deutscher Universitäten. Teil III. In: Berichte zur Wissenschaftsgeschichte 11, 105-117. 
Bornhak, Conrad (1900): Geschichte der preussischen Universitätsverwaltung bis 1810. Berlin: Georg Reimer.

Brinker, Klaus (2000): Textfunktionale Analyse. In: Klaus Brinker et al. (Hrsg.), Text- und Gesprächslinguistik (Handbücher zur Sprach- und Kommunikationswissenschaft 16). 1. Halbbd. Berlin, New York: de Gruyter, 175-186.

Bruning, Jens (2011): Vorlesungsverzeichnisse. In: Ulrich Rasche (Hrsg.), Quellen zur frühneuzeitlichen Universitätsgeschichte. Typen, Bestände, Forschungsperspektiven (Wolfenbütteler Forschungen 128). Wiesbaden: Harrasowitz, 269-292.

Bruning, Jens (2012): Innovation in Forschung und Lehre. Die Philosophische Fakultät der Universität Helmstedt in der Frühaufklärung 1680-1740 (Wolfenbütteler Forschungen 132). Wiesbaden: Harrasowitz.

Clark, William (2006): Academic Charisma and the Origins of the Research University. Chicago, London: University of Chicago Press.

Cod. Aug. = Lünig, Johann Christian (1724): Codex Augusteus oder neuvermehrtes Corpus juris Saxonici, worinnen die in dem Churfürstenthum Sachsen und darzu gehörigen Landen, auch denen Marggrafthümern Ober- und Nieder-Lausitz, publicirte und ergangene Constitutiones, Decisiones, Mandata und Verordnungen enthalten [...] Leipzig: Johann Friedrich Gleditsch Sohn.

Dinkel, Thilo (2000): Universitäts-Programmata als personengeschichtliche Quellen. In: Zeitschrift für württembergische Landesgeschichte 59, 427-431.

Döring, Detlef (1996): Samuel Pufendorf. Briefwechsel (Samuel Pufendorf. Gesammelte Werke, Bd. 1). Berlin: Akademie-Verlag.

Döring, Detlef (2002): Die Geschichte der Deutschen Gesellschaft in Leipzig. Von der Gründung bis in die ersten Jahre des Seniorats Johann Christoph Gottscheds (Frühe Neuzeit 70). Tübingen: Niemeyer.

Döring, Detlef (2009): Anfänge der modernen Wissenschaften. Die Universität Leipzig vom Zeitalter der Aufklärung bis zur Universitätsreform 1650-1830/31. In: Enno Bünz, Manfred Rudersdorf \& Detlef Döring (Hrsg.), Geschichte der Universität Leipzig 1409-2009. Bd. 1: Spätes Mittelalter und Frühe Neuzeit. Leipzig: Leipziger Universitätsverlag, 521-771.

Döring, Eduard (1837): Handbuch der Münz- und Wechselkunde. Frankfurt/M.: Hermann. Doyé, Sabine (2012): Das Eherecht der deutschen Frühaufklärung im Spiegel des neuzeitlichen Naturrechts. Hugo Grotius, Samuel Pufendorf, Christian Thomasius, Christian Wolff. In: Marion Heinz \& Sabine Doyé (Hrsg.), Geschlechterordnung und Staat. Legitimationsfiguren der politischen Philosophie (1600-1850). Berlin: AkademieVerlag, 57-117.

DTA = Deutsches Textarchiv. Online: www.deutschestextarchiv.de (03.02. 2018).

Düffel, Peter v. (Hrsg.) (1970): Christian Thomasius. Deutsche Schriften. Stuttgart: Reclam.

${ }^{1}$ DWB $=$ Deutsches Wörterbuch von Jacob Grimm und Wilhelm Grimm. Bd. I-XVI. Leipzig: Hirzel 1854-1960. Quellenverzeichnis 1971.

Fandrych, Christian \& Maria Thurmair (2011): Textsorten im Deutschen. Linguistische Analysen aus sprachdidaktischer Sicht (Stauffenburg Linguistik 57). Tübingen: Stauffenburg.

[Fingerlos, Matthäus] (1798): Ueber öffentliche Lehranstalten insbesondere über Lektionskataloge auf Universitäten. Erstes Bändchen. Germanien.

Fleischmann, Max (1929): Christian Thomasius. Rede zur 200. Wiederkehr von Thomasius' Todestag (23. Sept. 1728) gehalten bei der Gedenkfeier der Vereinigten FriedrichsUniversität Halle-Wittenberg (Hallische Universitätsreden 39). Halle/S.: Niemeyer.

Forssmann, Knut (1977): Balthasar Gracian und die deutsche Literatur zwischen Barock und Aufklärung. Diss. Mainz. Barcelona. 
Füssel, Marian (2015): Lehre ohne Forschung? Zu den Praktiken des Wissens an der Universität der Frühen Neuzeit. In: Martin Kintzinger \& Sita Steckel (Hrsg.), Akademische Wissenskulturen. Praktiken des Lehrens und Forschens vom Mittelalter bis zur Moderne (Veröffentlichungen der Gesellschaft für Universitäts- und Wissenschaftsgeschichte 13). Basel: Schwabe, 59-87.

Fulda, Daniel (2008): Von der Polyhistorie zur modernen Wissenschaft. Zum politischgalanten Gelehrtenideal der Frühaufklärung. In: Ulrich Johannes Schneider (Hrsg.), Kulturen des Wissens im 18. Jahrhundert. Berlin, New York: de Gruyter, 281-288.

Grotefend, Hermann (1891-1898): Zeitrechnung des deutschen Mittelalters und der Neuzeit. 2 Bde. Hannover: Hahn.

Gundling, Nicolaus Hieronymus (1744): Allgemeines Geistliches Recht der drey Christlichen Haupt-Religionen [...]. Anderer und letzter Band. Frankfurt, Leipzig: Wolfgang Ludwig Spring.

Hasse, Ernst (1885): Geschichte der Leipziger Messen. Leipzig: Hirzel.

Hausendorf, Heiko, Wolfgang Kesselheim, Hiloko Kato \& Martina Breitholz (2017): Textkommunikation. Ein textlinguistischer Neuansatz zur Theorie und Empirie der Kommunikation mit und durch Schrift (Reihe Germanistische Linguistik 308). Berlin, Boston: de Gruyter

Heerde, Hans-Joachim \& Ulrich Joost (2006): Einleitung. In: Hans-Joachim Heerde, Das Publikum der Physik. Lichtenbergs Hörer (Lichtenberg-Studien XIV). Göttingen: Wallstein, 11-54.

Heinemann, Wolfgang (2008): Textpragmatische und kommunikative Ansätze. In: Nina Janich (Hrsg.), Textlinguistik. 15 Einführungen. Tübingen: Narr.

Hofmeister, A[dolph] (1887): Zum Thomasjubiläum. In: Die Grenzboten 46/4, $294 \mathrm{f}$.

Horn, E[wald] (1897): Kolleg und Honorar. Ein Beitrag zur Verfassungsgeschichte deutscher Universitäten. München: Academischer Verlag.

Huttner, Markus (2007a): Vorlesungsverzeichnisse als historische Quelle. Zu Entstehungsgeschichte, Überlieferungslage und Aussagewert Leipziger Lektionskataloge vom 17. zum 19. Jahrhundert. Wiederabdruck in: Ders., Gesammelte Studien zur Zeit- und Universitätsgeschichte. Münster: Aschendorff, 189-218.

Huttner, Markus (2007b): Geschichte als akademische Disziplin. Historische Studien und historisches Studium an der Universität Leipzig vom 16. bis zum 19. Jahrhundert. Aus dem Nachlaß herausgegeben von Ulrich von Hehl (BLUWiG, Reihe A, Bd. 5). Leipzig: Evangelische Verlagsanstalt.

Jones, William Jervis (1976): A Lexicon of French Borrowings in the German Vocabulary (15751648) (Studia Linguistica Germanica 12). Berlin, New York: de Gruyter.

Kämper, Heidrun (1999): Art. ,Diskurs‘. In: Deutsches Fremdwörterbuch. 2. Aufl. Bd. 4. Berlin, New York: de Gruyter, 669-675.

Kalender A = Daisigner, Johann Christoph [1657]: Der Grosse Alt und Newe Schreib-Calender/ auff das Saturnini-Jovialische Haupt- nach der heilsamen Geburt JEsu Christi. M. DC. LVIII. [...] Leipzig: Ritzschen.

Kalender B = Schneider, Bartholomaeus [1678]: Alter und Neuer Haußhaltungs- und Geschicht-Calender. Auffs Jahr nach der heiligen Geburt unsers HErrn JEsu CHristi M. DC. LXXIX. Leipzig: Ritzsch.

Kalender C = Lindner, Friedrich [1680]: Alt- und Neuer Leipziger Haupt- und GeschichtCalender Auff das Jahr nach JESU Gebuhrt M. DC. LXXXI. Leipzig: Katharina Fiebig. Kalender D = [Junius, Ulrich] [1699]: Verbesserter Calender Auf das Jahr 1700. Leipzig: Thomas Fritsch. 
Kalender E = [Junius, Ulrich] [1700]: Practica, Oder gewöhlicher Anhang Des Calenders/ Darinnen von den vier Zeiten des Jahrs/ ihren Witterungen/ [...] und allerhand andern nützlichen/ und dem gemeinen Mann dienlichen Sachen/ so in diesem Jahr Christi 1701. Zu bedencken fürfallen/ umständlich gehandelt wird. [Leipzig: Thomas Fritsch].

Kretzenbacher, Heinz Leonhard (1990): Rekapitulation. Textstrategien der Zusammenfassung von wissenschaftlichen Fachtexten (Forum für Fachsprachen-Forschung 11). Tübingen: Narr.

Le Cam, Jean-Luc (2016): Vorlesungszettel und akademische Programme. Zur Rekonstruktion des akademischen Betriebs und Lebens jenseits der Lektionskataloge: das Beispiel des Helmstedter Rhetorikprofessors Christoph Schrader (Professur 1635-1680). In: Jan-Hendryk de Boer, Marian Füssel \& Jana Madlen Schütte (Hrsg.), Zwischen Konflikt und Kooperation. Praktiken der europäischen Gelehrtenkultur (12.-17. Jahrhundert) (Historische Forschungen 114). Berlin: Duncker \& Humblot, 89-137.

Lee, Seung-Kee et al. (Hrsg.) (2012): Philosophical Academic Programs of the German Enlightenment. Stuttgart-Bad Cannstatt: Frommann-Holzboog.

Lehmstedt, Mark (1992): Weidmann und Thomasius. Dokumente zur Leipziger Buchgeschichte des späten 17. Jahrhunderts. In: Leipziger Jahrbuch zur Buchgeschichte 2, 327-372.

Lieberwirth, Rolf (1955): Christian Thomasius. Sein wissenschaftliches Lebenswerk. Eine Bibliographie (Thomasiana 2). Weimar: Hermann Böhlaus Nachfolger.

Longo, Mario (2012): Welcher Gestalt man denen Frantzosen in gemeinem Leben und Wandel nachahmen solle? In: Seung-Kee Lee et al. (Hrsg.), Philosophical academic programs of the German enlightenment. A literary genre recontextualized (Forschungen und Materialien zur Universitätsgeschichte 1/4). Stuttgart-Bad Cannstatt: FrommannHolzboog, 3-13.

Luden, Heinrich (1805): Christian Thomasius, nach seinen Schicksalen und Schriften dargestellt. Berlin: Unger.

Luig, Klaus (1999): Art. ,Osse, Melchior von‘. In: Historische Kommission bei der Bayerischen Akademie der Wissenschaften (Hrsg.), Neue Deutsche Biographie. Bd. 19. Berlin: Duncker \& Humblot, $609 \mathrm{f}$.

Marquart, Heinz (1977): Matthäus Fingerlos (1748-1817). Leben und Wirken eines Pastoraltheologen und Seminarregenten in der Aufklärungszeit. Göttingen: Vandenhoeck \& Ruprecht.

Marti, Hanspeter (2011): Dissertationen. In: Ulrich Rasche (Hrsg.), Quellen zur frühneuzeitlichen Universitätsgeschichte. Typen, Bestände, Forschungsperspektiven (Wolfenbütteler Forschungen 128). Wiesbaden: Harrasowitz, 293-311.

Marti, Hanspeter (2013): Rez. von: Lee, Seung-Kee et al. (Hrsg.): Philosophical academic programs of the German enlightenment. A literary genre recontextualized, StuttgartBad Cannstatt 2012. In: Informationsmittel (IFB) 21/4. Online: http://ifb.bsz-bw.de/ bsz377446424rez-1.pdf (03. 02. 2018).

Müller, Gerhard (2001): Die extraordinäre Universität - Jenas Modernisierungsweg. In: Gerhard Müller, Klaus Ries \& Paul Ziche (Hrsg.), Die Universität Jena. Tradition und Innovation um 1800 (Pallas Athene 2). Stuttgart: Steiner, 191-196.

Münkler, Herfried (2015): Kriegssplitter. Die Evolution der Gewalt im 20. und 21. Jahrhundert. Berlin: Rowohlt.

Neumann, F[lorian] (2005): Art. ,Programm‘. In: Gert Ueding (Hrsg.), Historisches Wörterbuch der Rhetorik. Bd. 7. Tübingen: Niemeyer, Sp. 154-158.

Neuper, Horst (2003): Das Vorlesungsangebot an der Universität Jena von 1749 bis 1854.

2 Bde. Weimar: VDG. 
Neuß, Erich (1931): Christian Thomasius' Beziehungen zur Stadt Halle. In: Max Fleischmann (Hrsg.), Christian Thomasius. Leben und Lebenswerk (Beiträge zur Geschichte der Universität Halle-Wittenberg 2). Halle/S.: Niemeyer, 453-478.

Oberhausen, Michael \& Riccardo Pozzo (Hrsg.) (1999): Vorlesungsverzeichnisse der Universität Königsberg (1720-1804) (Forschungen und Materialien zur Universitätsgeschichte 1/1). 2 Bde. Stuttgart-Bad Canstatt: Frommann-Holzboog.

Opel, Julius Otto (Hrsg.) (1894): Christian Thomasius. Kleine deutsche Schriften. In: Festschrift der Commission der Provinz Sachsen zur Jubelfeier der Universität HalleWittenberg. Halle/S.: Otto Hendel.

Paulsen, Friedrich \& Rudolf Lehmann (1919-1921): Geschichte des gelehrten Unterrichts auf den deutschen Schulen und Universitäten vom Ausgang des Mittelalters bis zur Gegenwart. 3., erw. Aufl. 2 Bde. Leipzig: Veit.

Pörksen, Uwe (1986): Deutsche Naturwissenschaftssprachen. Historische und kritische Studien (Forum für Fachsprachen-Forschung 2). Tübingen: Narr.

Pörksen, Uwe (1990): Ist die Sprache ein selbständiger Faktor der Wissenschaftsgeschichte? (Akademie der Wissenschaften und der Literatur Mainz, Abhandlungen der Klasse der Literatur 1990, Nr.2). Wiederabdruck in: Ders. (1994), Wissenschaftssprache und Sprachkritik. Untersuchungen zu Geschichte und Gegenwart (Forum für FachsprachenForschung 22). Tübingen: Narr, 17-35.

Pohl, Anja (2016): Studentisches Leben an der Universität Leipzig im Zeitalter der Aufklärung. Diss. Leipzig. Online: urn:nbn:de:bsz:15-qucosa-219161 (03. 02. 2018).

Polenz, Peter v. (1994): Deutsche Sprachgeschichte vom Spätmittelalter bis zur Gegenwart. Bd. 2. Berlin, New York: de Gruyter.

Pozzo, Riccardo (1999): Vorlesungsverzeichnisse als Quelle der Universitätsgeschichte Preußens. In: Reinhard Brandt \& Werner Euler (Hrsg.), Studien zur Entwicklung preußischer Universitäten (Wolfenbütteler Forschungen 88). Wiesbaden: Harrasowitz, 59-80.

Pozzo, Riccardo (2012): Introduction. In: Seung-Kee Lee u. a. (Hrsg.), Philosophical academic programs of the German enlightenment. A literary genre recontextualized (Forschungen und Materialien zur Universitätsgeschichte 1/4). Stuttgart-Bad Cannstatt: FrommannHolzboog, IX-XVIII.

Prinz, Michael (2019): Sprachwahl und Heldenmythos - Deutsch als historische Wissenschaftssprache zwischen Popularisierung und Skandalisierung (erscheint 2019).

Rasche, Ulrich (2008): Über Jenaer Vorlesungsverzeichnisse des 16. bis 19. Jahrhunderts. In: Thomas Bach, Jonas Maatsch \& Ulrich Rasche (Hrsg.), „Gelehrte“ Wissenschaft. Das Vorlesungsprogramm der Universität Jena um 1800 (Pallas Athene 26). Stuttgart: Franz Steiner, 13-57.

Rasche, Ulrich (2009a): Seit wann und warum gibt es Vorlesungsverzeichnisse an den deutschen Universitäten? In: Zeitschrift für Historische Forschung 36, 445-478.

Rasche, Ulrich (2009b): Art. ,Privatdozent'. In: Friedrich Jaeger (Hrsg.), Enzyklopädie der Neuzeit. Bd. 10. Stuttgart: Metzler, 360-363.

Reichel, Gottlieb (1742): Thränen und Seuffzer wegen der Universität Leipzig denen getreuen Land Ständen geoffenbaret. Hrsg. von Georg Witkowski. Leipzig: Reclam. 1929.

Rolf, Eckard (1993): Die Funktionen der Gebrauchstextsorten (Grundlagen der Kommunikation und Kognition). Berlin, New York: de Gruyter.

Rolf, Eckard (2000): Textuelle Grundfunktionen. In: Klaus Brinker et al. (Hrsg.), Text- und Gesprächslinguistik (Handbücher zur Sprach- und Kommunikationswissenschaft 16). 1. Halbbd. Berlin, New York: de Gruyter, 422-435.

[Rotth, Albrecht Christian] (1692): Doppelte Verthäidigung Des Eben=Bildes Der Pietisterey. Freyburg. 
Rüegg, Walter (Hrsg.) (1996): Von der Reformation zur Französischen Revolution (1500-1800) (Geschichte der Universität in Europa II). München: Beck.

Ruge, Arnold (1840): Die Panegyrik der Orthodoxie und die Kritik unserer Tage. In: Hallische Jahrbücher für Wissenschaft und Kunst 3, Sp. 1905-1908.

Sauer, August (1894): Vorbemerkung. In: Ders. (Hrsg.), Christian Thomasius. Von der Nachahmung der Franzosen. Nach den Ausgaben von 1687 und 1701 (Deutsche Litteraturdenkmale des 18. und 19. Jahrhunderts N.F. 51). Stuttgart: Göschen'sche Verlagshandlung, III-IX.

Sauter, Johann Leonhard (1686): L' Homme De Cour Oder Balthasar Gracians Vollkommener iStaats- und Welt-Weise/ Mit Chur-Sächsischer Freyheit. Leipzig: Adam Gottfried Kromayer.

Schneider, Ulrich Johannes (1999): Philosophie und Universität. Historisierung der Vernunft im 19. Jahrhundert. Hamburg: Meiner.

Schneiders, Werner (Hrsg.) (1993): Introductio ad philosophiam aulicam (Christian Thomasius, Ausgewählte Werke, Bd. 1). Hildesheim u. a.: Olms.

Schrader, Wilhelm (1894): Geschichte der Friedrichs-Universität zu Halle. Bde. 1-2. Berlin: Dümmler.

Schröder, Konrad (2017): Sprachmeister des Französischen, Italienischen und Englischen in der Stadt und an der Universität Halle im 18. Jahrhundert. Versuch einer Bestandsaufnahme, mit einem Exkurs zum Hallenser Polnischen Lektorat. In: Max Häberlein \& Holger Zaunstöck (Hrsg.), Halle als Zentrum der Mehrsprachigkeit im langen 18. Jahrhundert (Hallesche Forschungen 47). Halle: Franckesche Stiftungen, 37-61.

Schröpfer, Horst (2003): Kants Weg in die Öffentlichkeit. Christian Gottfried Schütz als Wegbereiter der kritischen Philosophie (FMDA II/18). Stuttgart-Bad Cannstatt: Frommann-Holzboog.

Steinberg, Georg (2005): Christian Thomasius als Naturrechtslehrer (Hallesche Schriften zum Recht 22). Köln u. a.: Carl Heymanns.

Steinberg, Georg (Hrsg.) (2010): Programmata Thomasiana, et alia scripta similia breviora coniunctim edita (Christian Thomasius, Ausgewählte Werke, Bd. 21). Hildesheim u. a.: Olms.

Stollberg-Rilinger, Barbara (2000): Europa im Jahrhundert der Aufklärung. Stuttgart: Reclam. Tütken, Johannes (2005): Privatdozenten im Schatten der Georgia Augusta. Zur älteren

Privatdozentur (1734 bis 1831). Teil I: Statutenrecht und Alltagspraxis. Göttingen: Universitätsverlag.

VD 17 = Das Verzeichnis der im deutschen Sprachraum erschienenen Drucke des 17. Jahrhunderts. Online: www.vd17.de (03. 02. 2018).

Weißbach [= Selintes], Christian (1711): Balthasar Gracians, Homme De Cour, Oder: Kluger Hof- und Welt-Mann/ Nach Monsieur Amelot de la Houssaie, Seiner Frantzösischen Version, ins Teutsche übersetzet/ Von Selintes. Nebst Ihro Excellenz des Königl. Preussischen Herrn Geheimbten Raths/ Christiani Thomasii, Judicio vom Gracian. Augsburg: Paul Kühtzen.

Weißenborn, Bernhard (1931): Die Bibliothek des Christian Thomasius. In: Max Fleischmann (Hrsg.), Christian Thomasius. Leben und Lebenswerk (Beiträge zur Geschichte der Universität Halle-Wittenberg 2). Halle/S.: Niemeyer, 421-452.

Wille, v., Dagmar (1991): Lessico filosofico della „Frühaufklärung“. Christian Thomasius, Christian Wolff, Johann Georg Walch (Lessico Intellectuale Europeo LIV). Rom.

Zedler $=$ Zedler, Johann Heinrich (1731-1754): Grosses vollständiges Universallexicon aller Wissenschafften und Künste. 64 Bde. Leipzig, Halle: Zedler.

Zenne, Karl-Heinz (1955): Christian Thomasius-Ausstellung 1955. In: Wissenschaftliche Zeitschrift der Martin-Luther-Universität Halle-Wittenberg. Gesellschafts- und sprachwissenschaftliche Reihe 4/4, 565-568. 